\title{
Elternschaft und Gender Trouble
}

\section{Inszenierungen moderner und tradierter Mutterbilder}

\author{
HELGA KRÜGER-KIRN \& LEILA ZOË TICHY \\ UNTER MitARBEIT VON ANNA ELSÄSSER
}

\section{EinLeitung}

Die Fallstudie Mutterschaft und Geschlechterverhältnisse im Projekt REVERSE untersucht vor dem Hintergrund antifeministischer Entwicklungen in der Gesellschaft den gegenwärtigen Diskurs um Familie und Mutterschaft. Auf der Grundlage einer diskursanalytischen Analyse von populären Elternzeitschriften sowie Interviews mit Müttern werden insbesondere die Themen ,Vereinbarkeit ${ }^{1}{ }^{1}$ von Erwerbsund Familienarbeit, Mutter-Kind-Bindung und vergeschlechtlichte Elternrollen in den Blick genommen. Erkenntnisleitend für die Studie ist dabei die Frage, inwiefern sich hier ein Bezug zu aktuellen antifeministischen Debatten herausarbeiten lässt und welche Konsequenz und gesellschaftspolitische Funktion die Anschlussstellen an antifeministische Vorstellungen von Mutterschaft und Familie haben.

Ausgangspunkt unserer Fallstudie ist die Beobachtung widersprüchlicher kultureller Deutungsmuster von Mutterschaft, Vaterschaft und Elternschaft. Gleichstellungspolitiken steht ein Beharren auf traditionellen Geschlechter- und Familienstrukturen (Bundesinstitut für Bevölkerungsforschung 2017) gegenüber. Diese werden durch eine Zuspitzung antifeministischer Argumentationen und Skandalisierungen verstärkt, mit der die Figur der Mutter als Zentrum der vermeintlich bedrohten Kleinfamilie in den Fokus rückt. Beiden Polen gemeinsam ist ein - wenn auch

1 Wir setzen, Vereinbarkeit' bewusst in Anführungszeichen, da diese Formulierung die kategorische Unterscheidung der jeweiligen Sphären bereits voraussetzt und auf diese Weise ,normal' erscheinen lässt. Die Markierung dient dem Verweis und der Reflexion auf die dem Kapitalismus immanente Unterscheidung von Reproduktionsarbeit und produktiver Arbeit. 
sehr unterschiedlicher - Bezug auf Mutterschaft und Familie. Im ersten Fall werden emanzipatorische Errungenschaften mit der Berufstätigkeit der Mutter sowie der Pluralisierung von Familienkonstellationen verknüpft, im zweiten Fall werden Mutterbilder aufgerufen, die die Mutter als zentrale Figur für eine gelingende Entwicklung des Kindes im Rahmen der Familie adressieren und dies mit alltagspsychologischen Vorstellungen über die kindliche Entwicklung untermauern (Diabaté 2015).

Der Fallstudie liegt die Annahme zugrunde, dass es insbesondere dort zu Überschneidungen gleichstellungsorientierter und traditioneller Geschlechterdiskurse kommt, wo es um familiäre Strukturen und das sogenannte Wohl der Kinder geht. Zudem gehen wir davon aus, dass sich antifeministische Argumentationen nicht nur in jenen Kontexten finden lassen, die sich explizit gegen die Pluralisierung und Entnormierung geschlechtlicher und sexueller Lebensweisen richten, sondern auch in Begründungszusammenhängen rund um Familie, insbesondere zu Fragen der ,Vereinbarkeit" und der Mutter-Kind-Beziehung. Für unsere Fallstudie ist dabei von Belang, dass sich die Grenzlinien zwischen Antifeminismus und Feminismus oft nicht klar und deutlich ziehen lassen, sondern Zurückweisungen feministischer Forderungen mit positiven Bezugnahmen auf Emanzipation und Gleichstellung koexistieren (Klaus 2016). Unsere eigene Perspektive auf Antifeminismus geht von einem breiten, auf Herrschaftskritik ausgerichteten Feminismus-Verständnis aus und fasst Antifeminismus als weltanschauliche Gegenbewegung hierzu auf (vgl. die Beiträge von Henninger und Birsl in diesem Band). Diese Perspektive dient uns als Analyseinstrument für diskursive und gesellschaftliche Entwicklungen. Entsprechend steht in unserer Fallstudie in Bezug auf die Analyse elterlicher Positionen nicht nur das Verständnis von vergeschlechtlichten Elternpositionen und Familie im Fokus, sondern auch der Bezug zu geschlechterpolitischen Strukturen der Gesellschaft.

Vor diesem Hintergrund legt die Fallstudie Mutterschaft und Geschlechterverhältnisse den Fokus auf populärmediale Diskurse zu Mutterschaft und analysiert, inwieweit in den untersuchten medialen Diskursen zur ,Vereinbarkeit ${ }^{\star}$ von Erwerbs- und Familienarbeit, zur Mutter-Kind-Beziehung und zu vergeschlechtlichten Elternrollen Argumentationen enthalten sind, die Mutterschaft sowie die Position der Mutter im familialen Gefüge an den Erhalt von binären elterlichen Geschlechterkonstruktionen knüpfen und sie von Beginn an in ein geschlechterhierarchisches Verhältnis setzen.

Die hierfür durchgeführte Diskursanalyse bezieht sich auf einen Korpus von Artikeln aus den Zeitschriften Nido, Baby und Familie und Eltern aus den Jahren 2010 bis 2017. Die analytische Rekonstruktion der darin erkennbar werdenden Anforderungen an Mutterschaft und Familie soll dazu beitragen, eventuelle Diskursverschiebungen in Bezug auf Elternschaft und ,Vereinbarkeit' zu erfassen, um so das Verständnis für mögliche Andockstellen an antifeministische Argumentationen zu erweitern. Im Anschluss an die Diskursanalyse wurden leitfadengestützte Interviews mit Müttern von kleinen Kindern durchgeführt; die Interviewpartnerinnen 
wurden nach ihren subjektiven Erfahrungen zu den genannten Themenbereichen befragt. Hier fokussierten Interview-Fragen und Auswertung darauf, inwiefern Mütter sich an normativen Diskursen und Anrufungen orientieren, ob sie diese explizit problematisieren bzw. auch krisenhafte Erfahrungen artikulieren.

In unserem Beitrag stellen wir die Befunde aus beiden Teilen der Untersuchung vor und loten in einem Fazit aus, welche Verschränkungen zwischen antifeministischen Argumentationen und den von uns analysierten Diskursen bestehen und wie diese Befunde in den Gesamtkontext des Forschungsprojekts zu Antifeminismus eingebettet werden können.

\section{ZEITDIAGNOSEN UND AKTUELLER FORSCHUNGSSTAND}

Mit dem Wandel hin zu einer „flexibilisierten Geschlechterordnung“ (Lenz 2013: 212) haben sich im Zuge von Gleichstellungsbewegungen gesellschaftlich anerkannte Institutionen wie die Kleinfamilie (inklusive des damit oft verknüpften Ernährer-/Hausfrauenmodells) sowie der Wohlfahrtstaat verändert - und damit auch die strukturellen Anforderungen an Reproduktionsarbeit. Im Zweiten Gleichstellungsbericht der Bundesregierung (BMFSFJ 2017) wird der ,Gender-Care-Gap` benannt und ein ,Erwerb-und-Sorge-Modell' als (neue) Orientierung für politische Maßnahmen angeführt. Ziel hierbei ist es, Männern* wie Frauen*2 gleichermaßen Erwerbsarbeit, und parallel dazu auch Familienarbeit, zu ermöglichen (BMFSFJ 2017). In der Familienpolitik realisiert sich diese Ausrichtung einerseits im Ausbau der institutionellen Kindertagesbetreuung und von Ganztagsschulen, andererseits in der Einführung des Elterngeldgesetzes mit Lohnersatzfunktion (2007) und im 2008 reformierten Unterhaltsrecht. Nach Althammer ist das Elterngeld „,[d]er wesentliche Bruch mit dem gerechtigkeitsorientierten Verständnis staatlicher Familienpolitik“ (Althammer 2009: 163f, zit. nach Menke 2017: 45), da mit dem neuen, am individuellen Einkommen orientierten Elterngeld vom vorherigen Prinzip der Umverteilung zugunsten einkommensschwacher Eltern abgewichen wurde.

Der familienpolitische Wandel zum sogenannten Adult-Worker-Modell stellt nach Lessenich (2008: 17) eine Aktivierung der Bürger*innen für den Arbeitsmarkt ins Zentrum. Dieser Paradigmenwechsel geht mit einem Wandel im kulturellen Leitbild einher, das sich zunehmend auf „Eigenverantwortung“ stützt (Derboven

2 Als Hinweis auf den Konstruktionscharakter von Männern* und Frauen* nutzen wird das Zeichen *. Diese Bezeichnung schließt auch transsexuelle und genderqueere Menschen ein, die ebenfalls Erfahrungen mit Mutterschaft haben. Wir formulieren unsere Überlegungen mit Bezug auf die gegenwärtige symbolische Ordnung der Zweigeschlechtlichkeit. (Rede von Männern und Frauen). 
2019). Obwohl sich wesentliche Maßnahmen in der Gleichstellungs- und Familienpolitik auf die ,Vereinbarkeit' von Familie und Beruf beziehen und inzwischen von vielen Paaren eine egalitäre Elternschaft angestrebt wird, lässt sich eine (Re-)Traditionalisierung der Aufgabenbereiche feststellen, sobald ein Kind geboren wird. Zahlreiche Studien belegen, dass sich die Geschlechterverhältnisse und -ungleichheiten innerhalb der Familien mit Kindern kaum verändert haben (Maierhofer/ Strasser 2016; BMFSFJ 2012). Der Anteil erwerbstätiger Frauen* ist zwar gestiegen, aber immer noch wenden Mütter die meiste Zeit für unbezahlte Familienarbeit auf. Diese Struktur findet sich auch in der „modernisierten Versorger-Ehe“ als meist gelebtem Familienmodell wieder (Statistisches Bundesamt 2019). Zugleich zeigt der Siebte Familienbericht (BMFSFJ 2006), dass viele Familien unter sozioökonomisch prekären Bedingungen leben und insbesondere Mütter von Armut bedroht sind.

Mit der rechtlichen Anerkennung pluraler familiärer Lebensformen, etwa durch die 2017 eingeführte Möglichkeit zur Eheschließung für homosexuelle Paare (,Ehe für alle'), geht ein weiterer Paradigmenwechsel mit weitreichenden Konsequenzen für die normative Vormachtstellung der heterosexuellen Kleinfamilie einher. Mehr denn je steht die tradierte geschlechtliche ,Dreieinigkeit von Vater, Mutter, Kind“ auf dem Spiel und dies führt zu teilweise recht scharfen gesellschaftspolitischen Kontroversen. Auch antifeministische Diagnosen zum Wandel der Geschlechterordnung der modernen Gesellschaft werden mit dem angesprochenen Wandel von Familienbeziehungen in Verbindung gebracht. So konstatiert Scheele (2015) eine Verschiebung hin zu einem familienzentrierten Antifeminismus, in dem die heterosexuelle Kleinfamilie mit ihren vergeschlechtlichten Elternrollen eine zentrale Rolle spielt. In den entsprechenden antifeministischen Argumentationen wird ein eindeutiger Bruch gegenüber einer vermeintlich natürlichen zweigeschlechtlichen Familienstruktur unterstellt. Verantwortlich dafür sei die im Zuge von Emanzipationsprozessen veränderte Rolle der Frau*. Demgegenüber wird dann eine Position der ,Frau* als Mutter' eingefordert, die auf naturalistischen Vorstellungen von Geschlechterdifferenz beruht. Damit eng verbunden ist die Vorstellung einer eindeutigen Geschlechtsidentität (entweder weiblich/mütterlich oder männlich/väterlich), die auf biologischen Zusammenhängen gründet. Ungeachtet historischer Transformationen von Familienstrukturen und Elternpositionen wird über ein bestimmtes Bild von ,gelingendem Familienleben' eine patriarchale Ordnung der Geschlechter als ,natürlich“ imaginiert und zugleich romantisiert. Mit dem Ziel heterosexuelle Ordnungsmodelle und Genealogien zu stabilisieren, wird die hypostasierte Naturalisierung der Familie in den Dienst antifeministischer und auch nationalistischer Politiken gestellt (Kemper 2014; Lang 2017).

Einen weiteren prominenten Schauplatz rhetorischer Instrumentalisierung von Mutterschaft und Geschlechterverhältnissen stellt der Kindeswohldiskurs dar, der meist unmittelbar an den Vereinbarkeitsdiskurs geknüpft wird (Klaus 2016). Anti- 
feministische Formen der Bezugnahme auf Kinder als unschuldige und zu schützende Menschen (Schmincke 2015) fungieren hierbei als Projektionsfläche für affektive Mobilisierungen gegen Selbstbestimmungsrechte und -wünsche von Frauen*.

Wie Maihofer und Schutzbach (2015: 205) aufzeigen, zielen antifeministische Argumentationen nicht nur auf die Sicherstellung des ,,sogenannten natürlichen Zusammenhangs von Geschlecht und Mutterschaft und damit verbunden von Heterosexualität und Familie.“ Es wird zudem das Szenario entworfen, dass ,,mit der Vervielfältigung von Geschlecht und Sexualität“ die Auflösung der Familie einhergeht. Das wird auch deutlich in den Analysen von Kemper (2016) in Bezug auf die Familienpolitik rechtspopulistischer Parteien wie der AfD herausgearbeitet: Die exklusiv als weiß, bürgerlich und heterosexuell gedachte Kleinfamilie wird hier als „Keimzelle der Nation“ (AfD 2016: 40, zit. nach Kemper 2016: 3) und darüber hinaus explizit als „Identifikations- und Schutzraum“ für die in männlicher Form adressierten „Bürger“ entworfen (AfD 2016: 17, zit. nach Kemper 2016: 3). Die so gedachte „aktivierende Familienpolitik“ (AfD 2016: 41, zit. nach Kemper 2016: 5) nutzt rassistische Diskurse, aber auch Argumente der Lebensschutzbewegung sowie vermeintliche Erkenntnisse der Entwicklungspsychologie, um das Thema ,Familie ‘ an die heterosexuelle Kleinfamilie zu binden und eine Fremdbetreuung von Kindern zu diskreditieren (AfD 2016: 43, zit. nach Kemper 2016: 4). Die traditionellen Geschlechterverhältnisse sind in dieser Logik der elementare Kern des angestrebten Gesellschaftsmodells, weshalb sie unbedingt ,bewahrt' und vor der ,Gefährdung ‘ durch feministische Projekte und Gleichstellungspolitiken , geschützt` werden müssen.

Solche Begründungszusammenhänge zielen auf den Rückbau fundamentaler Frauen*rechte sowie auf den Erhalt der binären Geschlechterhierarchie und leisten somit Entwicklungen wie der Prekarisierung von Care-Arbeit oder mütterlicher Altersarmut Vorschub. Die Bundesarbeitsgemeinschaft kommunaler Frauenbüros und Gleichstellungsstellen (BAG 2018) wertet die Diffamierung der Gleichstellung von Mann* und Frau* allgemein als antidemokratisch und als Verstärkung einer antifeministischen und frauenverachtenden gesellschaftlichen Stimmung.

Angesichts der Entwicklung der politischen Diskurslandschaft der letzten Jahre lässt sich eine paradoxe Gleichzeitigkeit der Zurückweisung von feministischer Politik bei gleichzeitiger Bezugnahme auf diese feststellen. Gegenwärtig koexistieren einflussreiche zeitgenössische Diskurse zu ,Gender' mit neoliberaler Individualisierung und konservativ-biologistischen Rollenmodellen. McRobbie (2009) verortet diese Bewegungen in einem ,postfeministischen“ Diskurshorizont, der sich durch Argumentationsfiguren der Individualisierung und Selbstverantwortung sowie die Desartikulation politisch-struktureller Zusammenhänge auszeichnet. Dabei wird auf einen (Gleichstellungs-)Feminismus Bezug genommen, dessen zentrale Forderungen als bereits erreicht gelten. Diese Orientierung macht einen Feminismus, der 
sich auf gesamtgesellschaftliche Verhältnisse bezieht und einen grundlegenden Wandel der sozialen und symbolischen Ordnung anstrebt - bis hinein in die intimsten und vertrautesten Geschlechterbeziehungen (Gerhard 2012: 7) - anscheinend obsolet; sie verdeckt damit aber die nach wie vor bestehenden gesellschaftlichen Schieflagen (McRobbie 2009). Im Zusammenhang mit vergeschlechtlichten Familienvorstellungen und Fragen der ,Vereinbarkeit' stellt der Topos Mutterschaft, an der Schnittstelle von Individuum und Gesellschaft, einen bedeutsamen Knotenpunkt dar. Es verwundert daher nicht, dass sich unterschiedliche ideologische Diskurse gerade darauf richten.

\section{DiskuRsanALYSE DER ELTERNZEITSCHRIFTEN}

Elternzeitschriften, verstanden als special interest magazines, sind in Buchhandlungen und Kiosken beziehungsweise in Apotheken und Arztpraxen allgemein zugänglich. Sie können einerseits als Teil eines öffentlich-medialen Diskurses betrachtet werden, andererseits werden gezielt Eltern bzw. Mütter als Leser*innen adressiert und dementsprechende Themen verhandelt. In diskursanalytischer Perspektive stellen Elternzeitschriften somit ein Archiv dar, in dem implizites und explizites Wissen, Diskurse und Normen sichtbar werden. Allgemein erweckt die Textgestaltung der Artikel den Eindruck, dass Gleichberechtigung bzw. das Ideal von gleichberechtigter Elternschaft in den Zeitschriften angekommen sind. In der sprachlichen Adressierung von Eltern und Müttern fallen allerdings Widersprüche auf: Während in der Überschrift in der Regel zunächst von ,Eltern' oder auch ,Mutter oder Vater gesprochen wird, erfolgt im weiteren Verlauf häufig eine alleinige Zentrierung auf die ,Mutter', sei es im Text selbst oder anhand der ausgewählten Beispiele. ${ }^{3}$

\subsection{Empirischer und methodischer Zugang}

Um den medialen Diskurs um Mutterschaft im Hinblick auf die Themen, Vereinbarkeit" von Lohn- und Familienarbeit, Mutter-Kind-Bindung und vergeschlechtlichte Elternrollen zu untersuchen, wurden verschiedene Elternzeitschriften ausgewählt: Nido adressiert eine konsumstarke Mittelschicht (Auflage: 0,24 Millionen; AWA o.J. für 2017); Eltern (als traditionsreiche Zeitschrift seit 1966) richtet sich an ein breiteres Publikum (Auflage: 1,07 Millionen; AWA o.J. für 2018); Baby und Familie hat als kostenlose Apothekenzeitschrift die höchste Auflage (Auflage: 1,77 Millionen; AWA o.J. für 2018). Den Textkorpus für die Analyse bildeten insgesamt

3 Hierzu zählen z.B. Interviews und Bilder. Eine vertiefte Analyse medialer Mittel kann hier nicht geleistet werden. 
93 Artikel aus den Zeitschriften Nido, Eltern sowie Baby und Familie aus den Jahren 2010 bis 2017.

In Anlehnung an die Kritische Diskursanalyse von Siegfried Jäger (1993) und Margarete Jäger (2008) haben wir aus diesem Textkorpus Argumentationsfiguren, Diskursstränge, aber auch Stereotypien und Kollektivsymboliken herausgearbeitet. Angelehnt an die Forschungsfragen wurden drei Diskursstränge identifiziert: Der erste Strang bezieht sich auf die ,Vereinbarkeit‘ von Lohn- und Familienarbeit, der zweite auf die Konstruktion von vergeschlechtlichten Elternrollen und der dritte auf Konstruktionen der Mutter-Kind-Beziehung. Die Aufteilungen in Diskursstränge und Diskursfiguren sind dabei analytische Differenzierungen; in den Texten treten Diskurse nie in Reinform, sondern vermischt auf. So wird die Mutter-Kind-Beziehung insbesondere in Artikeln über Gesundheit und Entwicklung des Kindes sowie über Themen wie Stillen, Geburt und Wochenbett verhandelt; diese bilden in den Elternzeitschriften eine Art Grunddiskurs. Der Diskursstrang zum Thema, Vereinbarkeit' konnte u.a. anhand von Artikeln zum Thema, Wiedereinstieg in den Beruf' rekonstruiert werden. Die Unterschiedlichkeit der vergeschlechtlichten Elternrollen konnte insbesondere aus Artikeln herausgearbeitet werden, die die Arbeitsaufteilung unter den Eltern thematisieren sowie aus dem Diskurs um ,neue Vaterschaft', dem viele Artikel explizit gewidmet sind.

\subsection{Die Figur der Mutter zwischen modernen und konservativen Geschlechtskonstruktionen}

Im Zuge der Analyse der Mutterbilder sticht eine Rhetorik der ,Wahlfreiheit" und ,Machbarkeit‘ ins Auge, die die individuelle Verantwortung ins Zentrum rückt, ohne jedoch die Bedingungen, die diese ermöglichen oder verhindern, zu thematisieren. Inhaltlich bezieht sich der Diskurs auf die Gestaltung des Familienlebens und der Berufstätigkeit. Der Fokus auf die individuelle Verantwortung für die eigene Lebenssituation zeigt sich dabei an Formulierungen wie folgender: „Aber viele über Stress jammernde Arbeitnehmer haben es sich selbst ausgesucht." (Nido 10.2013) Auch in Bezug auf Tätigkeiten im Haushalt wird Überforderung nicht als Kritik oder Anlass für Veränderungen verstanden, sondern als ,Jammern` dargestellt. Diese Argumentation spitzt sich zu, wenn die Anlässe für Kritik in einer Weise hinterfragt werden, die die Mutter zur eigentlichen Urheberin der angesprochenen Probleme macht. So wird etwa gefordert, dass die Mutter ihre Sauberkeitsansprüche hinterfragen solle, wenn sie unzufrieden mit der Arbeitsteilung im Haushalt ist: „Fragen Sie sich: Muss es zu Hause immer picobello sauber sein?“ (Baby\&Familie 02.2017) $\mathrm{Zu}$ solch individualisierenden Argumenten kommen ,Tipps ‘ und Ratschläge hinzu, die ebenfalls den Eindruck erwecken, dass alles möglich sei, solange nur der Wille und die Einsatzbereitschaft stimmen. So wird Müttern beispielsweise 
beim Thema ,Vereinbarkeit` empfohlen, ihren Marktwert herauszustellen und die eigene Flexibilität und Produktivität zu betonen: „Werden Sie aktiv und präsentieren Sie Ihrem Arbeitgeber Lösungen, um ihm diese Sorgen zu nehmen - so früh wie möglich.“ (Baby\&Familie 02.2014) Die Verknüpfung von Selbstverantwortung mit Wahlfreiheit zeigt sich nicht nur in Bezug auf die ,Vereinbarkeit‘ von Beruf und Familie, sondern kommt auch in Bezug auf das Gelingen des Paar- und Familienlebens sowie die positive Entwicklung des Kindes zum Einsatz. Im Fokus stehen dabei stets das Handeln und Fühlen der Mütter als selbstverantwortliche Einzelpersonen und nicht die Kooperation als Elternpaar oder die Arbeitsbedingungen und -zeiten der Eltern sowie deren finanzielle Ressourcen.

Im Lichte dieser individualisierenden Rhetorik erscheinen die Herausforderungen der ,Vereinbarkeit' als reine Privatangelegenheit. Infolge dessen werden diesbezügliche Konflikte und Lösungen als individuelle Erfolge und/oder Beziehungsproblematiken des Paares verhandelt. Dazu bedarf es einer affektiven Politik (Gill 2016; Rutherford 2018), die durch die Regulierung von Gefühlen und die Arbeit am Selbst gekennzeichnet ist. Auf diese Weise kann der Anschein der Selbstgestaltung und Verantwortung gewahrt bleiben und lässt sich zugleich mit tradierten Vorstellungen einer ,guten Mutter` verknüpfen, wie in folgendem Zitat deutlich wird: „Eine gute Mutter sorgt für sich selbst.“ (Eltern 11.2016) Hier bietet das Narrativ, dass Frauen* als Mütter bereits autonome und ermächtigte Subjekte sind (Rutherford 2018; McRobbie 2009), gleichzeitig Anschlussstellen an tradierte heteronormative Vorstellungen von Mutterschaft. Dabei übernehmen die Argumentationsfiguren der Individualisierung sowie der Fokus auf Machbarkeit die Funktion, heteronormative Zuschreibungen an Eltern als selbstbestimmte individuelle Wahl einzukleiden. Die Mutter wird so im doppelten Sinne adressiert: einerseits (selbst-)verantwortlich und gleichzeitig selbstbestimmt, indem sie ihre Position als Mutter auf freien Entscheidungen gründet.

\subsection{Die Figur der Do-it-all Mother}

Für die Mutterfigur, die durch diese Anrufungen konstruiert wird, haben wir den Begriff „Do-it-all Mother“ vorgeschlagen (Tichy/Krüger-Kirn 2019). Die Do-it-all Mother erfüllt alle an sie gerichteten Anforderungen: Sie kommt ihren ,Pflichten als Mutter' als hauptverantwortliche Bezugsperson für das Kind nicht nur nach, sondern erfüllt ihre Mutterrolle auf professionelle Weise. Dabei ist sie beruflich erfolgreich und bleibt zugleich sowohl äußerlich attraktiv als auch emotional ausgeglichen. Eine Do-it-all Mother übernimmt die normativen Zuschreibungen an Mütter proaktiv: „Meine Prioriät im Alltag ist: dass ich alles selber mache: Kinder, Job und Haushalt." (Eltern 06.2015) Privilegien von finanziell besser gestellten Familien werden als eigene Leistung affirmiert und auf diese Weise strukturelle Verhältnisse 
ausgeblendet. Äußerungen von Kritik oder die Artikulation von Leidenserfahrungen werden als ,Jammern' verharmlost und in die Nähe von persönlicher (Charakter-) Schwäche gerückt. Die Kehrseite des individualisierenden Selbstmanagements sind Disziplinierungsanrufungen: „Meine Damen, machen sie sich locker“, konstatiert Nido zum Thema Überforderung mit Mutterschaft (Nido 09.2010). Zu diesem Themenkomplex gehört auch, grundlegende finanzielle und strukturelle Familienbedingungen sowie die damit einhergehenden Konflikte und Unvereinbarkeiten als Anforderung der ,Work-Life-Balance' zu kodieren. Auch hier wird allerdings fast ausschließlich die Mutter angesprochen. So wird einer gestressten Mutter z.B. empfohlen, „erst einmal in Ruhe einen Tee zu trinken“, bevor der Haushalt erledigt wird (Baby\&Familie 02.2017).

Anstelle einer Reflexion struktureller Bedingungen und sozioökonomischer Einflussfaktoren adressieren die dargestellten Diskursstränge über den Rekurs auf Achtsamkeit und Selbstfürsorge die emotionale und subjektive Verfasstheit der (Mutter-)Subjekte. Psychologische Interventionen zur emotionalen Stressbewältigung und Selbstregulation richten sich dabei nicht nur in erster Linie an Mütter, sondern schaffen auch die Voraussetzungen für gegenseitiges „mother blaming“ (Sommerfeld 1989; vgl. dazu auch Kap. 4.7).

Mit Blick auf den Diskursstrang der ,Vereinbarkeit" treten in den Zeitschriften weitere Ansprüche an die Figur der Mutter zutage. Obwohl diese durchgängig als primäre Bezugsperson für das Kind konstruiert wird, wird ein Wiedereinstieg in den Beruf nicht in Frage gestellt. Letzteres verknüpft sich allerdings mit Empfehlungen zur Teilzeitarbeit oder zu einem Stellenwechsel, evtl. auch zur beruflichen Selbstständigkeit, die als optimale Möglichkeit von ,mütterlicher Vereinbarkeit` beworben wird. Dies unterstreicht, dass die Verantwortung für das Gelingen der, Vereinbarkeit" bei der Mutter liegt. So wird in den Zeitschriften immer wieder betont, dass Mutter-Sein ohne Erwerbsarbeit ungenügend sei: „Müttergespräche reichen mir nicht.“ (Eltern 06.2015) Das ,Hausfrauenmodell‘ muss offenbar an die Modernisierung der Mutterrolle angepasst werden, die ja auch dem Bild einer, emanzipierten Frau*` entsprechen soll. Durch Rhetoriken der Professionalisierung wird die Mutterposition aufgewertet, und gleichzeitig werden konservative Geschlechterrollen und Familienkonzepte an den modernen Diskurs familiärer Selbstgestaltung anschlussfähig gemacht. Die Mutter als „Familienmanagerin“ (Eltern 06.2013) neben einem gutverdienenden Ehemann unterscheidet sich dann kaum noch von der erfolgreichen ,Karriere-Mutter'. Damit einhergehende potenzielle Widersprüchlichkeiten können über den Fokus auf individuelle Einstellungen scheinbar aufgelöst werden: „Es ist schwer, aber Mütter und Väter kriegen es trotzdem hin, weil sie es wollen.“ (Eltern 10.2015) Während die Rhetorik der Selbstbestimmung hier immerhin noch beide Elternteile adressiert, fällt die Verantwortung dann letztendlich doch wieder auf die Mutter zurück, denn: „Für die Stunden, die sie in der Arbeit verbringt, entscheidet sich eine Mutter dagegen beim Kind zu sein.“" (Eltern 04. 
2010) Auf diese Weise stellt sich die Berufstätigkeit der Mutter als individueller Selbstverwirklichungswunsch dar, dessen Konsequenzen sie folglich auch alleine zu verantworten hat.

Die wirtschaftliche Notwendigkeit zur Lohnarbeit als persönlichen Wunsch einer Mutter darzustellen, verdeutlicht, wie mütterliche Rollenvorstellungen - aktuell die Figur der Do-it-all Mother - dazu führen, dass strukturelle Bedingungen aus dem Blick geraten oder gar verdeckt werden. Sozioökonomische Aspekte, wie prekäre finanzielle Verhältnisse von Familien, aber auch eine Reflexion der Vaterposition, rücken in den Hintergrund. So können geschlechterkonservative Zuschreibungen an Eltern, insbesondere an Mütter unangetastet bleiben. Diese beziehen sich in erster Linie auf Rollenvorstellungen von Mutter und Vater, die entlang der Annahme der Geschlechterbinarität auf ein biologisches Verständnis von Mutterschaft und mütterlichem Körper rekurrieren.

\subsection{Der mütterliche Körper und der Kindeswohldiskurs}

Der Diskurs der Mutter-Kind-Bindung zeigt, dass die Mutter und ihre Tätigkeiten sowie ihre Beziehung zum Kind in erster Linie als hormongesteuerter Prozess vorgestellt werden. Die „Macht der Hormone“ (Baby\&Familie 07.2010) ,steuert“ schon die werdende Mutter, wenn etwa gefragt wird, ob eine Frau* durch die körperlich-hormonalen Prozesse im Zuge von Schwangerschaft und Geburt ,,auf Autopilot“" (Nido 07/08.2012) sei. Der Hormondiskurs reduziert das mütterliche Erleben und Verhalten auf biologische, scheinbar ,natürliche‘ Prozesse. Auf diese Weise wird nicht nur die Eigenaktivität der Mutter, eine Beziehung und Bindung zum Kind aufzubauen, auf die Biologie reduziert; dies dient zugleich der Konstruktion einer ,natürlichen“ Differenz zwischen Mutter- und Vaterrolle.

In diesem Sinne werden elterliche Tätigkeitsfelder und Zuständigkeiten für die Versorgung und die Beziehung zum Kind antagonistisch entworfen: Die Mutter als fürsorgliche, emotionale und vor allem konstant körperlich anwesende Figur symbolisiert den zentralen Ort der Fürsorge und Geborgenheit. Die Beziehung des Vaters zum Kind entwickelt sich hingegen nicht über Fürsorgetätigkeiten, sondern primär über Aktivitäten, die sich auf die Spiel- und Freizeitgestaltung beziehen. Hier treten stereotype Konzepte von Mutterschaft und Vaterschaft deutlich zu Tage, die den Handlungsspielraum sowohl für die Frau* als Mutter wie für den Mann* als Vater einschränken.

Ähnliche rhetorische Strategien lassen sich auch in Bezug auf den Diskursstrang ,Kindeswohl‘ herausarbeiten. Der dem Kindeswohldiskurs inhärente Anspruch , das Beste für das Kind' zu garantieren, geht mit zahlreichen normativ aufgeladenen Forderungen an Eltern, insbesondere an Mütter, einher. Damit das Kind stressresistent, gesund, intelligent und sozial werden kann, braucht es den ,sicheren Hafen“ 
der Familie: „Liebe macht gesund, sozial, stressresistent und schlau.“ (Baby\& Familie 12.2011) Das Kindeswohl wird dabei in einer Weise mit einer erfolgs- und leistungsorientierten Entwicklung des Kindes verknüpft, die nicht nur die Verantwortung dafür an die Figur der Mutter bindet, sondern über die ,Liebe“ auch an den mütterlichen Körper. Bezugnahmen auf die Bindungstheorie (Bowlby 1973) adressieren hier meist ausschließlich die Mutter, was weder dem Stand der Bindungsforschung (Marga 2013) noch der aktuellen Bandbreite von Familienkonstellationen entspricht.

Der elterliche Wunsch, dem eigenen Kind ein gesundes und glückliches Aufwachsen zu ermöglichen, wird allerdings nicht nur normativ funktionalisiert und gegendert. Die alleinige Adressierung der Mutter fokussiert - auch über Schwangerschaft, Geburt und Stillzeit hinausgehend - auf den mütterlichen Körper. Denn implizit ist mit den vergeschlechtlichten Lesarten der Bindungsforschung eine mütterliche Präsenz- und Verantwortungsverpflichtung verbunden, die vor dem Hintergrund der mütterlichen Berufstätigkeit auch als Kritik an der gesellschaftlichen Gleichstellungspolitik und Fremdbetreuung von Kindern gelesen werden kann. Dass Transformationen im Geschlechterverhältnis auch das elterliche Beziehungsverhältnis betreffen und für die Verantwortung für Kinder neue Formen und Rahmenbedingungen gefunden und entwickelt werden müssen, bleibt hier unthematisiert. Ebenso wenig wird die gesellschaftspolitische Verantwortung für ein gelingendes Aufwachsen zum Thema.

\subsection{Der ,neue Vater“ und die Geschlechterbinarität}

Der ,neue Vater' wird in den Zeitschriften als modernes Vorbild angepriesen und vielfach thematisiert. Auch hier wird die bereits mehrfach herausgearbeitete Begründungsfigur einer biologischen Binarität des heterosexuellen Elternpaars für die Unterschiedlichkeit der vergeschlechtlichten Elternrollen in Anschlag gebracht und als Zugewinn von Pluralität codiert. Wenn betont wird, „Papa macht's anders“ (Baby\&Familie 11.2017), wird die väterliche Position mit Rationalität und Strenge auf der einen und Spiel und Abenteuer auf der anderen Seite assoziiert. Auf traditionelle psychoanalytische Entwicklungsmodelle zurückgreifend, gewinnt der Vater als ,Befreier des Kindes aus der Mutter-Kind-Dyade‘ und als ,Begleiter des Kindes zur Außenwelt‘ (Metzger 2017) eine zentrale Bedeutung.

Trotz einer Reklamation väterlicher Autorität verkörpert der Vater die eher lustbetonten Seiten der Elternschaft, wodurch er auch sich selbst und das ,Kind im Mann*‘ entdecken kann. Die Rede vom ,romantische[n] Traum der aktiven Vaterschaft" (Baby\&Familie 11.2010) deutet neben einer Abgrenzung vom traditionellen autoritären Vaterbild zugleich an, dass Wunsch und Wirklichkeit weit auseinanderliegen (können). Zugleich können dann mit einer solchen Formulierung das fehlen- 
de Engagement von Vätern bei Haushalt und Kinderpflege verharmlost werden. Als Erklärung dafür wird zum einen das Fehlen von männlichen Vorbildern für junge Väter herangezogen, zum anderen die ,Übereifrigkeit‘ der Mutter. Die oben thematisierte Verpflichtung der Mutter auf Kinder und Haushalt fällt also doppelt auf die Mutter zurück, denn nun wird sie auch für das mangelnde väterliche Engagement bei reproduktiven Tätigkeiten verantwortlich gemacht. „Das Gelingen der VaterKind-Beziehung hängt entscheidend von der Mutter ab.“ (Baby\&Familie 11.2010)

Der Vater wird hier nicht nur als ,Verlierer' der Flexibilisierung und Gleichberechtigung der Geschlechter dargestellt; im Unterschied zur Mutter wird er in seiner Eigenverantwortung nicht ernst genommen, eher sogar infantilisiert. Dies lässt nicht nur auf implizit wirksame geschlechterstereotype Elternrollen, sondern auf ein grundlegendes ,sameness taboo ' schließen. Das Fehlen eines analogen Narrativs einer ,neuen Mutterschaft" verweist darauf, dass das Modell von ,neuer Vaterschaft ${ }^{\star}$ bisher nicht geschlechtergerecht zu Ende gedacht und auch (noch) nicht sozial anerkannt ist.

Ebenso wie in Analysen von Elternratgebern (z.B. Rinken 2012: 26) zeigt sich auch in den Elternzeitschriften, wie durch den Diskurs um ,neue Vaterschaft' ${ }^{6}$ zwar der Handlungsspielraum von Vätern erweitert wird, die Verpflichtungen der Mütter jedoch weitgehend die gleichen bleiben - oder sogar noch ausgeweitet werden. Letztlich wird in der Untersuchung des Diskursstranges der vergeschlechtlichten Elternrollen deutlich, dass der Bezug auf Differenzen zugleich einer Zementierung und erneuten Herstellung von Ungleichheit und Hierarchie zwischen Mutter und Vater dient. Die in den Zeitschriften dominanten Deutungsmuster mütterlicher und väterlicher Positionen können damit fast nahtlos an patriarchale Familienkonstellationen anschließen. Zwar lässt sich eine Erweiterung des Mutterbildes um Entscheidungsfreiheit, Selbstverantwortung und Berufstätigkeit feststellen, allerdings wird die grundlegende Zuweisung der Fähigkeit zu bemuttern an die Mutter nicht in Frage gestellt, ebenso wenig wie ihre Verantwortung für die Entwicklung des Kindes und ein gelingendes Familienleben. So bleiben trotz der häufigen Rede von ,neuer Vaterschaft' die Rollen von Mutter und Vater geschlechtsstereotyp gebunden. Unsere Ergebnisse stimmen mit anderen Untersuchungen überein, die zeigen, wie über eine Rhetorik von Wahlfreiheit letztlich vergeschlechtlichte Elternpositionen reproduziert werden (McRobbie 2009: 43, 81). Auf diese Weise wird Geschlecht nur noch implizit als Ordnungsprinzip sichtbar. Moderne Konzepte wie der ,neue Vater' oder die Do-it-all Mother können so ungestört mit konservativen oder traditionellen Geschlechterrollen in der Elternschaft koexistieren. 


\subsection{Die Unsichtbarmachung von „queerer Differenz“}

Vordergründig vermitteln die untersuchten Zeitschriften durchaus den Eindruck, sexuelle Vielfalt sowie eine Vielfalt von Familienformen und Elternkonstellationen positiv zu würdigen, obwohl es eine starke Unterrepräsentanz von nicht heterosexuellen, alleinerziehenden, gering verdienenden und nicht-weißen Eltern gibt. Auf Grundlage dieser Beobachtung und im Hinblick auf die Frage der Diskursivierung unterschiedlicher Familienformen wurde eine Feinanalyse von zwei Artikeln über lesbische Elternschaft durchgeführt. Dabei konnte gezeigt werden, dass lesbische Elternschaft über die Strategie der ,affirmativen Assimilation“ (Tichy/Krüger-Kirn 2020) zwar positiv markiert wird, zugleich allerdings an das Modell der heterosexuellen Kleinfamilie mit ihren binären Elternpositionen angepasst wird.

Das Konzept ,Familie“ gewinnt auch hier zentrale Bedeutung und wird ebenso wie bei heterosexuellen Elternpaaren als Ort angerufen, an dem eine gesunde, kindliche Entwicklung stattfinden kann und soll. Diese Perspektive auf Familie setzt nicht unbedingt einen leiblichen Vater voraus, wohl aber geschlechterbinäre Elternrollen. In den beiden Artikeln zu lesbischer Elternschaft zeigt sich, dass die Position des Vaters flexibel besetzt werden kann, indem entweder ein „weiblicher Papa“ (Eltern 11.2014) oder eine zweite Mutter zu der angenommenen Mutter-Kind-Dyade hinzukommt: „Du kommst nicht aus meinem Bauch - und bist trotzdem mein Baby! Lesbische Frauen, die ihr Kind nicht selbst geboren haben, entwickeln schnell eine ganz eigene Art von Mutterliebe.“ (Eltern 11.2014) Hier nimmt die biologische Mutter eine vergleichbare Position ein wie in heterosexuellen Familienkonstellationen.

Auch das Narrativ der Kleinfamilie als Sehnsuchtsort und Ort der (entsexualisierten) Liebe kann offenbar unabhängig von geschlechtlicher Orientierung angeeignet werden. Über den Fokus auf Liebe und die individuelle Liebesgeschichte als zentrale Aspekte von Familie treten geschlechtliche und strukturelle Ungleichheiten und Diskriminierungen - die teilweise in den Artikeln durchaus benannt werden in den Hintergrund. Daher sprechen wir von affirmativer Assimilation und schließen an kritische Debatten darüber an, wie über Strategien der Normalisierung homosexuelle Paare an den herrschenden Diskurs gebunden werden und dabei die Sichtbarkeit der Homosexualität der Individuen verloren geht (Smith 1994; Adam 1995).

Zusammenfassend zeigt die Dynamik der affirmativen Assimilation, wie eine Anerkennung homosexueller Elternschaft möglich ist, allerdings um den Preis der Unsichtbarmachung von geschlechtlich-sexuellen Emanzipationsentwicklungen und „queerer Differenz“ (Walters 2012: 919). Anstelle einer Problematisierung bestehender Ungerechtigkeiten in den Geschlechterverhältnissen tritt ein Fokus auf wählbare Möglichkeiten und die Betonung des gesellschaftlichen Fortschritts, der 
sich darin ausdrückt, Familie auf vielfältige Weise leben zu können. Gerade in Verbindung mit der neoliberalen Rhetorik der Wahlfreiheit kann affirmative Assimilation als diskursive Bewältigungsstrategie gedeutet werden, um Gender und Geschlechterdifferenz so in Einklang zu bringen, dass die heterosexuelle Grundordnung von Elternschaft und Familie nicht in Frage gestellt wird. Einerseits nivelliert die augenscheinliche Akzeptanz geschlechtlicher Vielfalt in den Elternkonstellationen und ihre Einhegung in den heteronormativen Diskurs ihre potenzielle Sprengkraft für die heterosexuelle Grundordnung. Andererseits zeigt die Strategie der affirmativen Assimilation deutlich, dass mit homosexuellen Familienkonstellationen eine potenzielle Gefährdung der bestehenden ,harmonischen' Ordnung (Fröhlich/ Hellwig/Spicker 2018: 12) einhergeht.

\subsection{Die Verwendung von Differenzen zur Zementierung und Herstellung von Ungleichheit}

Während die Zeitschriften vordergründig den Eindruck vermitteln, die Vielfalt der Familienformen und Elternkonstellationen positiv zu bewerten, lässt sich trotz einer Anerkennung des gesellschaftlichen Wandels die normative Wirkmacht der heterosexuellen Kleinfamilie nachzeichnen (vgl. auch Thiessen/Villa 2009; Koppetsch/ Speck 2015). Als zentrales Ergebnis zeigt unsere Analyse der Zeitschriftentexte, wie über die Bezugnahme auf Selbstbestimmung und Gleichberechtigung sowie Selbstverantwortung und Wahlfreiheit konservative Vorstellungen und Positionen von Mutterschaft tradiert werden. Dabei stellt die Rhetorik der Wahlfreiheit wohl die entscheidende Schnittstelle dar, an der geschlechterstereotype Anrufungen an Mütter zugleich in einen postfeministischen Diskurshorizont der Selbstverantwortung und Individualisierung gestellt werden.

Die über alle Diskursstränge hinweg widersprüchliche Komplexität der MutterFigur wurde mit dem Begriff der „Do-it-all Mother“ (Tichy/Krüger-Kirn 2019) markiert. Das „Do-it-all“ repräsentiert dabei sowohl mütterliche Anrufungen und selbst gesetzte Ziele als auch die Funktion, die der Mutter als Zentrum und Ankerpunkt der Kleinfamilie zugeschrieben wird. Der Rekurs auf bestimmte Lesarten des Kindeswohls, der Mutter-Kind-Beziehung sowie auf die Naturalisierung der Mutterrolle zeigt, wie es diskursiv gelingt, tradierte Mutterrollen und Wahlfreiheit gleichzeitig zu affirmieren.

Auch wenn die Diskurse um ,Vereinbarkeit‘ und den ,neuen Vater' veränderte Handlungsspielräume und elterliche Beziehungsverhältnisse nahelegen, kann das emanzipative Potenzial veränderter Vorstellungen von Elternschaft durch die Verknüpfung mit geschlechterbinären Rollenzuweisungen kaum sichtbar und handlungsleitend werden. Die in ihnen wirksamen tradierten Geschlechtervorstellungen bestimmen nach wie vor eine geschlechterdifferente Arbeitsteilung der Eltern mit 
entsprechenden Mutter- und Vaterrollen (vgl. auch Dolderer et al. 2016; KrügerKirn 2017). Daran schließt die Diskursfigur der „affirmativen Assimilation“ (Tichy/Krüger-Kirn 2020) an, mittels derer homosexuelle Formen von Elternschaft vor dem Hintergrund klassischer Familienstrukturen bewertet und an heterosexuelle Familienstrukturen anschlussfähig gemacht werden; gleichzeitig dient diese Diskursfigur dazu, Pluralisierung zu feiern. „Queere Differenz“ (Walters 2012) bleibt dabei insofern unsichtbar, als eine Assimilation homosexueller Elternpaare an das Konzept der heteronormativen Kleinfamilie dominiert und letztere hierdurch stabilisiert wird.

Mit Bezug auf die Ausgangsfrage, ob es einen Zusammenhang zwischen Antifeminismus und modernen mütterlichen Weiblichkeits- und Familienbildern gibt, lässt sich im Rahmen der bisherigen Analyse festhalten, dass der Rekurs auf den Ort der Familie einschließlich vergeschlechtlichter Elternbilder einen entscheidenden gemeinsamen Referenzrahmen beider Diskurse darstellt. Eine weitere Parallele besteht darin, dass Familie zugleich den Ort der bestmöglichen kindlichen Entwicklung und Zugehörigkeit repräsentiert

Die hier gekennzeichneten Zusammenhänge und Dynamiken werden durch Vorstellungen vom Kindeswohl in ganz besonderer Weise gestützt. Darunter werden unterschiedliche Perspektiven auf die kindliche Entwicklung subsumiert, ohne allerdings die aktuelle wissenschaftliche Bandbreite zu berücksichtigen und als solche zu kennzeichnen. Die Ergebnisse der Bindungsforschung (Bowlby 1973, 1975) setzen das Bindungsbedürfnis des Kindes unabhängig von Geschlecht mit der Feinfühligkeit der Bezugsperson in Bezug (Marga 2013). Von der Position des Kindes ausgehend wird hierbei ein Zusammenhang von sicherer Bindung, explorativem Verhalten und affektiv-emotionaler Entwicklung aufgezeigt. Dagegen legen vergeschlechtlichte Lesarten der Bindungstheorie in den Zeitschriften den Eindruck nahe, dass Bindungssicherheit und eine stabile emotionale Entwicklung exklusiv an die leibliche Mutter gebunden sind, der die notwendigen Fähigkeiten wie Feinfühligkeit, Liebe und Empathie, von Natur aus' zugeschrieben werden. Hier lassen sich Querverbindungen zu antifeministischen Vertreter*innen identifizieren, die - oft unter Rekurs auf die Wissenschaft - diskriminierende Standpunkte gegenüber einer emanzipatorischen Gestaltung der Gesellschaft in Anschlag bringen (vgl. KrügerKirn et al. 2019).

Die in den Diskurssträngen herausgearbeiteten Perspektiven auf die Bedeutung der Biologie für die vergeschlechtlichten Mutter- und Vaterpositionen sowie die Mutter-Kind-Beziehung scheinen sich gegenseitig zu stabilisieren. Vor allem gelingt es dadurch, die Position der Mutter zu naturalisieren und über alle Familienformen hinweg konstant zu halten. Durch die weitgehende Ausblendung des historisch-gesellschaftlichen Kontextes wird der Eindruck unterstrichen, dass die Position der Mutter im familiären Gefüge ihrer ,Natur' entspricht. Auf diese Weise kann auch die Figur der ,guten Mutter ${ }^{6}$ als Konstante unangetastet bleiben. Auch wenn 
um sie herum Pluralität affirmiert werden kann, ist die Position der Mutter selbst offenbar nicht verhandelbar.

Während im antifeministischen Diskurs Angriffe auf Gender einen zentralen Bestandteil ausmachen, lässt sich dies für unsere Analyse der Artikel in Elternzeitschriften nicht bestätigen. Eher wird - ganz im postfeministischen Sinne - deutlich, dass am Anspruch einer Gleichberechtigung der Geschlechter festgehalten wird; dieser jedoch implizit über die Positionierung der Mutter im familiären Gefüge und über Bedeutungszuweisungen an die Mutterrolle unterlaufen wird. Die Abwehr einer emanzipatorischen Geschlechter- und Sexualitätspolitik offenbart sich hier also nicht in einem expliziten Antifeminismus, sondern eher in verdeckter Weise, indem über affirmative Assimilation eine Eingemeindung in heterosexuelle Familienbilder und damit in traditionell-bürgerliche Geschlechterverhältnisse erfolgt.

\section{INTERVIEWS MIT MÜTTERN}

Anknüpfend an die Analyse des medialen Diskurses um Mutterschaft in den Elternzeitschriften werden im Folgenden Ergebnisse aus den Interviews mit Müttern vorgestellt. Dabei wird gezeigt, welche Begründungszusammenhänge und Strategien wirksam werden, wenn es um das eigene Selbstverständnis als Mutter in Bezug auf die Themen ,Vereinbarkeit‘, ,Mutterrolle` und, innerfamiliäre Arbeitsteilung' geht.

\subsection{Methodischer und empirischer Zugang}

Es wurden insgesamt 17 leitfadengestützte Interviews mit Müttern geführt und inhaltsanalytisch ausgewertet. Die Interviews fanden zum großen Teil face to face statt, in Ausnahmen per Telefon, und dauerten jeweils circa eine Stunde. Die Gesprächspartner*innen hatten zwischen ein und vier Kindern, von denen das jüngste zwischen acht Monaten und vier Jahren alt war. In der Mehrzahl waren sie heterosexuell orientiert und in einer Partnerschaft lebend (11), eine war alleinerziehend, eine lebte in einem Co-Parenting-Modell und vier lebten in einer Beziehung mit gleichgeschlechtlicher oder transsexueller Partnerin. Einige Befragte studierten (5), die restlichen waren erwerbstätig. Alle Mütter hatten Elternzeit genommen, einige waren während des Interviews gerade in Elternzeit. Durch die Zusammensetzung der Gruppe sollte eine möglichst heterogene Stichprobe entstehen.

Alle Interviews wurden transkribiert, inhaltlich codiert und in Interpretationsgruppen ausgewertet. Während wir mit der kritischen Diskursanalyse versucht haben, Muster in den Diskursen zur ,Vereinbarkeit', zu vergeschlechtlichten Elternrollen und zur Mutter-Kind-Beziehung herauszuarbeiten und diese im diskursiven Kontext zu verorten, liegt der Fokus in der Analyse der Interviews auf der Verwo- 
benheit zwischen Diskurs und subjektiven Aneignungs- und Umgangsformen. Als individuelle Subjektivierungsweisen geben sie nicht nur Einblick in die Wirkmächtigkeit dominanter Diskurse, sondern auch in damit verbundene psychische Verarbeitungsprozesse.

Zur Strukturierung und inhaltlichen Differenzierung der Interviews wurde induktiv ein Klassifikationssystem mit zwei Ebenen entworfen. Als erste Ebene arbeiteten wir übergreifende Begründungszusammenhänge heraus, die sich latent und manifest in den Interviews finden lassen. ,Begründungszusammenhänge‘ beziehen sich auf den kontingenten Hintergrund von Aussagen und fungieren als implizite Voraussetzungen, wie beispielsweise die Geschlechterbinarität, mit der die verschiedenen Zuschreibungen an Vater- und Mutterrolle geordnet werden. Mit der zweiten Ebene, die wir hier als ,Strategien“ kennzeichnen, analysieren wir, wie diese Begründungszusammenhänge jeweils subjektiv zur Erklärung des eigenen Handelns eingesetzt werden. Da sich die Spannung zwischen hegemonialer Ordnung und ihrem Einfluss auf die Werte und Gefühle des Subjektes v.a. in latenten Sinnzusammenhängen und psychischen Umgangsweisen realisiert, wird diese AnalyseAchse durch einen psychoanalytischen Zugang vertieft. Psychische Wirkmechanismen werden dabei nicht nur auf der Ebene individueller Besonderheiten reflektiert, sondern in den Kontext zugrundeliegender - familialer und gesellschaftlicher - Strukturen gestellt. Eine psychoanalytische Perspektive auf den affektiven Charakter im Umgang mit Ambivalenz und Konflikthaftigkeit dekonstruiert z.B. individuelle Strategien des othering nicht nur als projektiven Mechanismus, der eine grundsätzliche Form der psychischen Verarbeitung darstellt, auf die Menschen zurückgreifen, wenn sie Identitätsverunsicherungen oder Ängste erleben. Weiterführend ist hier zudem, herauszuarbeiten, in welchen Situationen und Diskurskontexten dieser Mechanismus aktiviert wird.

Im Folgenden stellen wir nun die Ergebnisse der Interview-Analysen zu den Themen ,Vereinbarkeit', Vergeschlechtlichung der Elternrollen sowie Mutter-KindBeziehung dar.

\section{2 ,Vereinbarkeit' zwischen Gleichheitsnorm und Geschlechterbinarität}

Der Diskurs der Selbstverantwortung und Wahlfreiheit in Bezug auf die Herausforderungen, die mit ,Vereinbarkeit‘ einhergehen, spielt auch in den Interviews eine zentrale Rolle, und zwar sowohl als Begründungszusammenhang als auch als Strategie. Deutlich machen lässt sich dies an dem Anspruch der Mütter , alles selbstständig zu schaffen‘. Dies korrespondiert mit der in der Zeitschriftenanalyse herausgearbeiteten Diskursfigur der Do-it-all Mother: „Also für mich jetzt wäre überhaupt nicht in Frage gekommen zu sagen: ,Boar, ich finde das so anstrengend, 
kannst du bitte daheimbleiben? Ich gehe lieber arbeiten?‘ //mhm// ((verneinend)) mhm. Sondern für mich war klar, ich bin Mutter und ich muss es durchziehen.“ (Linda, Absatz 11) Arbeiten zu gehen, damit ihr Partner die Elternzeit und damit einen großen Teil der Familienarbeit übernehmen kann, würde Linda als mütterliches Versagen erleben. Mit ihrer Erklärung ,ich bin Mutter und ich muss es durchziehen" unterstreicht sie nicht nur ihre Identifikation mit dem Anspruch, die Erwartungen der Mutterrolle gut zu erfüllen und nicht ,aufzugeben', sondern richtet diesen explizit an sich als Mutter.

Hier bietet der implizite Begründungszusammenhang der Geschlechterbinarität einen vertieften Zugang, um zu verstehen, wieso der Anspruch des „Do-it-all“ gegendert und nur für die Mutter und nicht den Vater in Anschlag gebracht wird. Nicht in allen Interviews wird der Zusammenhang zwischen Geschlecht und normativen Erwartungen in Bezug auf die Elternrollen so explizit herausgestellt wie bei Linda. Er wird in vielen Interviews interessanterweise durch die Gleichheitsnorm verdeckt. Dass Geschlechterbinarität auch hier dennoch latent wirksam ist, wird ersichtlich, wenn beispielsweise Barbara die geschlechterstereotype Aufgabenaufteilung in Bezug auf Kinder und Haushalt über individuelle Vorlieben und Interessen begründet:

„Aber naja er sagt dann, er geht halt nicht so gerne nicht zum Kinderturnen oder zum ElternKind-Kreis, kann ich ja auch verstehen, ist er vielleicht auch nicht der Typ dafür. Mir macht das ja im gewissen Sinne auch schon Spaß, aber ja, das ist etwas, was ich einfach so schlucken muss, dann einfach. Das ist tatsächlich jetzt einfach so.“ (Barbara, Absatz 70)

Indem Barbara erklärt, dass ihr Mann* „nicht der Typ dafür“ sei, versucht sie die geschlechtliche Rollenaufteilung auf seine Individualität zu beziehen und damit zugleich zu rechtfertigen, warum ihr Mann* die benannten Aufgaben nicht übernimmt. Darüber gelingt es ihr auch, unterschiedliche Maßstäbe an sich und ihren Partner anzulegen. Während für ihren Partner individuelle Vorlieben ein Kriterium dafür darstellen, welche Aufgaben in der Familie übernommen werden, trifft das für Barbara nicht zu; im Gegenteil - sie muss diese Aufgabe „einfach so schlucken“. In diesem Sinne wirkt ihre Begründung, dass ihr diese Kurse „,in gewissem Sinne auch schon Spaß" machen, eher als Selbstbeschwichtigung; sie verdeutlicht, dass in der Aufgabenaufteilung Stereotypien wirksam sind, die über das Geschlecht der Eltern organisiert werden. Über den Bezug auf Individualisierung gelingt es hier, die geschlechterbinären Umgangsweisen und die geschlechtsstereotype Aufgabenwahl zu verdecken.

Der explizite Begründungszusammenhang der Gleichheitsnorm, der in diesem Interview eine starke Rolle spielt, gerät so mit dem implizit oder latent wirksamen Begründungszusammenhang der Geschlechterbinarität in Konflikt. Eine häufig verwendete Strategie, den Konflikt zwischen der vergeschlechtlichen Selbstver- 
ständlichkeit der familiären Position, hier der Mutterposition, und der Gleichheitsnorm zu ,lösen‘, erfolgt über den Begründungszusammenhang der Wahlfreiheit. Dieser Logik folgend erscheint die Wahl der elterlichen Position , genau so' gewollt und ,jederzeit umkehrbar'. Die Aufgabenteilung in der Familie erscheint damit grundsätzlich verhandelbar. In diesem Sinne argumentiert Barbara: „Oach, wenn ich jetzt gesagt hätte, ich will jetzt irgendwie auf jeden Fall arbeiten gehen oder irgendwie, dann hätte er bestimmt jetzt nicht nein gesagt“ (Barbara: Absatz 50). In dieser Perspektive der individuell wählbaren Rollenverteilung bleiben ihre Bedürfnisse und Wünsche gleichermaßen respektiert. Dazu führt Barbara weiter aus:

„[A]ber also ich wollte gerne zuhause bleiben und der hat auch also eine Arbeit, die er unglaublich gerne macht und ich war auch ganz froh, eine Auszeit zu haben tatsächlich. Also ich war auch ganz dankbar, dass ich jetzt damit eigentlich also, ne? ich wollte das eigentlich auch machen, hatte Lust da drauf. Ja.“ (Barbara: Absatz 50)

Trotz des expliziten Rekurses auf den Begründungszusammenhang der Wahlfreiheit deuten sich durch die Einschübe „eigentlich“ und ,auch“ Ambivalenzen an. Dabei macht die positive Hervorhebung der Erwerbsarbeit des Partners auf einen weiteren brisanten Punkt im Rahmen der Vereinbarkeitsanforderungen aufmerksam: auf die Hierarchisierung von Erwerbsarbeit und Familienarbeit, bzw. produktiver und reproduktiver Arbeit. Erst vor dieser Hierarchisierungsfolie kann die Elternzeit als ,Auszeit' codiert und bewertet werden, für die die Mutter dem Partner dann auch „dankbar" sein kann.

Zusammenfassend zeigen diese Interviewpassagen, dass der manifeste Begründungszusammenhang der Gleichheitsnorm gleichzeitig mit dem latent wirksamen Begründungszusammenhang der Geschlechterbinarität auftritt. Der mit der Gleichheitsnorm aufgerufene Individualisierungsdiskurs gewinnt strategische Wirkung, um geschlechterstereotype Tendenzen als individuelle Präferenzen und selbstverantwortete Wahl zu interpretieren. Das Ideal der Gleichheit erschwert somit, dass Geschlecht als Erklärung respektive Begründung für die familiäre Arbeitsteilung explizit sichtbar und reflektiert werden kann.

Mit Bezug auf Interviews, in denen der, Druck, dem Anspruch an eine moderne Frau* und Mutter zu genügen' thematisiert wird, soll nun im Folgenden der $\mathrm{Zu}-$ sammenhang des mütterlichen Selbstwerts mit Berufstätigkeit erhellt werden.

\section{3 ,Vereinbarkeit' und Selbstwert}

Auffällig ist der Versuch der Interviewpartnerinnen, sich von sogenannten Hausfrauen abzugrenzen und die Erwerbsarbeit als bedeutsamen Teil ihrer Identität zu begründen: „Mir war das immer wichtig, Kinder zu haben, aber eben auch immer 
wichtig, ein eigenes Berufsleben entwickeln zu können. Ne, dass man-, ich wollte nie Hausfrau sein und zuhause bleiben mit Kindern." (Lara, Absatz 8) Der Selbstentwurf als berufstätige Mutter wird hier eng mit dem Selbstwert verbunden. Lara geht in ihrer Elternzeit ihrer Selbstständigkeit nach und kann sich darüber als moderne berufstätige Mutter verstehen, auch wenn ihr eigenes Einkommen nicht ausreicht, um die Familie zu ernähren.

Andere Interviewpassagen verdeutlichen, dass ein Scheitern am Anspruch berufstätig zu sein, zu Selbstwertproblemen führen kann. Darauf macht Ramona aufmerksam, wenn sie beschreibt, dass sie unter Selbstwertkonflikten leidet, da sie während Studium und Elternzeit über kein eigenes Einkommen verfügt: „Weil jetzt bin ich halt von meinem Partner abhängig. Ne? Also ich verdiene immer noch kein eigenes Geld und das ist schon, das nagt an mir so oder das nagt an meinem Bild von, wie ich eigentlich, ja, also meinem Frauenbild auch, ne? Also, dass ich da nicht unabhängig sein kann, gerade. Ja.“ (Ramona, Absatz 12) Für Ramona ist die Berufstätigkeit der Weg zur finanziellen Unabhängigkeit. Obwohl sie den Umstand, dass die Familienarbeit nicht entlohnt wird, im Laufe des Interviews als ungerecht kritisiert, beschreibt sie zugleich einen inneren Konflikt zwischen ihrem Selbstbild als Frau* und ihrer Mutterrolle, der sich nicht nur auf die finanzielle Abhängigkeit vom Partner beziehen lässt, sondern auch auf einen Identitätskonflikt zwischen emanzipierten und traditionellen Mutterschaftspositionen.

Dieser Selbstwertkonflikt zeigt sich nicht nur bei in heterosexuellen Paarbeziehungen lebenden Müttern, sondern auch bei lesbischen Müttern. So will Bettina Familienarbeit eigentlich nicht abwerten, kämpft aber zugleich mit dem Gefühl, dass diese weniger wert ist:

"Sie geht arbeiten und verdient das Geld und ich gehe arbeiten und bleibe zuhause und kucke aber, dass irgendwie hier diese Dinge alle laufen. Aber das ist für mich selber, also ich will jetzt nicht sagen, dass es für mich selber keinen Wert hat oder einen geminderten Wert hat, aber ich weiß gar nicht, wie ich das sagen soll.“ (Bettina: Absatz 62)

Die darauf folgende Passage verdeutlicht, dass die Unsicherheit über den Wert ihrer Arbeit mit der fehlenden Bezahlung familiärer Arbeit zusammen hängt: „Und es ist schon so ein Gefühl, ob MICH jemand dafür bezahlt, dass ich das tue, weil die Kinder nicht nur für die kleine Familie, sondern eben auch für unsere Gesellschaft wichtig sind und unsere Welt, oder ob sozusagen meine Frau mich für das bezahlt, was ich hier tue." (Bettina: Absatz 66)

Eine Möglichkeit diesen Konflikt zu lösen, besteht in der argumentativen Aufwertung von Familienarbeit, z.B. über eine sprachliche Anlehnung an die Berufswelt. So codiert Annika Familienarbeit nicht als „Auszeit“ (wie Barbara), sondern explizit als ,Arbeit‘. Das ermöglicht, sowohl finanzielle Abhängigkeit wie geschlechterstereotype Aufgabenverteilung hinter einem professionalisierten und 
ökonomisierten Sprachduktus zu verbergen. Diese Strategie erfüllt zugleich den Zweck, die ,gewählte' Rolle als selbstbestimmte und selbstbewusste Wahl zu legitimieren:

„Es ist im Grunde wie ein Job, den ich komplett in Vollzeit ausführe. Und da gehört das komplette Management, Haushaltsmanagement, ich sage immer, ich leite die Haushaltsabteilung, die Babyabteilung, die Klamottenabteilung. Ich bin sozusagen die Leiterin dieses Privaten zurzeit. Auch zu $100 \%$. Und er ist beruflich, genau, da zu $100 \%$. Und das ist schon zurzeit eben sehr getrennt.“ (Annika, Absatz 46)

Annika begreift ihre Rolle als Mutter als einen Vollzeitjob, der verschiedene Aufgabenbereiche umfasst, Expertise verlangt und einer Leitungsfunktion gleichkommt. Sie stellt damit ihre Mutterrolle mit der Erwerbsarbeit ihres Mannes* auf die gleiche Ebene. Andererseits erlaubt diese gleichberechtigte Gegenüberstellung auch hier eine Legitimation der traditionellen Rollenaufteilung zwischen Mutter und Vater.

Dies markiert die beschriebene Ambivalenz zwischen einer Aufwertung von Mutterrolle und Familienarbeit und der Legitimation und Verdeckung von Geschlechterhierarchien. Diese Verdeckung hierarchischer und nicht-individueller Strukturen trägt gleichzeitig dazu bei, dass Mütter Probleme mit ,Vereinbarkeit' als individuelles Versagen verstehen und damit ihren Selbstwertkonflikt verstärken. Das eigene Selbstwertgefühl als Mutter ist sehr eng mit gesellschaftlichen Formen von Anerkennung und finanzieller Entlohnung verwoben. Vor diesem Hintergrund ist es naheliegend, dass sich die Thematik der Vereinbarkeit von Erwerbs- und Familienarbeit bei den interviewten Müttern zu einem Identitätskonflikt zwischen, guter Mutter' und ,emanzipierter Frau*' ausweitet, der eng mit Fragen des Selbstwerts in Verbindung steht.

\subsection{Umgang mit Normen}

Schon in den bisher analysierten Interviewpassagen deutete sich ein Konfliktfeld zwischen widersprüchlichen und unerreichbaren Normen an. Dass es hierbei nicht nur um Ambivalenzen, sondern um spannungsreiche Konflikte geht, lässt sich an Textstellen konkretisieren, in denen die Mütter nachträglich ihre Anpassung an Normen reflektieren und hinterfragen. Ein Beispiel liefert Barbara: „Genau, es kam schon, kam schon aus mir heraus, aber tatsächlich denke ich, dass es aus mir heraus kam, weil ich unglaublichen Druck schon von außen erlebt habe, war es mir wichtig, das alles zu machen und gar nicht alles aus meiner eigenen Überzeugung“ (Barbara, Absatz 36). Barbaras Überlegungen, ihre vermeintlich selbst gesetzten Normen von Mutterschaft jetzt differenzierter als ,unglaublichen Druck von außen“ zu 
betrachten, reflektiert ihre Identifikation mit gesellschaftlichen Erwartungen: „Ich bin eine erwachsene Frau und jetzt kriegen wir das zweite Kind und jetzt wird alles spitze und ich kann das alles schaffen und naja, was man so erwartet oder irgendwie in der Gesellschaft gerade so von so einer Frau, ne?, die ein Kind bekommt." (Barbara, Absatz 16) Ein dominierender fordernder Blick auf das Selbst verknüpft Erfolg und den gesellschaftlichen Druck der Machbarkeit mit der Vorstellung einer erwachsenen Frau* als Mutter. Schuldgefühle, Versagensängste und Selbstwertkonflikte treten so an die Stelle der Erkenntnis, dass bestimmte Erfahrungen Ausdruck nachvollziehbarer Überforderungen und damit legitim sind. Dieser Umgang mit Normen realisiert eine Kehrseite des Diskurses der Wahlfreiheit und richtet den Blick auf Strategien, die die Konflikte als selbstverschuldet interpretieren und so auf paradoxe Weise an die Figur der Do-it-all Mother anschlussfähig machen.

Selbst wenn Mütter von Erfahrungen berichten, in denen die Erwartungen explizit im Außen verortet werden, so erfolgt das nicht ohne Konflikte. Dies lässt sich beispielsweise mit Aussagen von Ramona illustrieren, die die Betreuungsfrage als Konflikt zwischen Selbstbestimmung und normativer Anpassung erlebt: „So die Stimmen aus unserem Umfeld waren da so da und haben irgendwie so gesagt, naja, so klein und irgendwie erst so ein Jahr alt und jetzt schon so lange, und dass ich da nicht irgendwie sagen wollte, jetzt geht sie noch bis um drei, nicht nur bis um zwei (in die Betreuung).“ (Ramona, Absatz 34) Obwohl Ramona die „Stimmen“ aus ihrem Umfeld eindeutig als normative Vorstellungen über das Kindeswohl identifiziert, löst sie ihr Unbehagen, indem sie sich zurückzieht. Eine selbstbewusste Positionierung scheint ihr durch die normative Aufladung des Kindeswohldiskurses nicht möglich.

Erwartungen von außen markiert auch Annika, wenn sie von dem Vorhandensein von „Knack-Punkten“ (Annika, Absatz 74) berichtet, an denen normative Vorgaben für gute Mutterschaft hängen. Dies zeichnet Annika beispielhaft am Umgang mit dem Stillen nach:

„Es gibt häufig eben, genau, so einen bestimmten Knack-Punkt. Wenn man den überschreitet, dann ist das nicht mehr cool, zum Beispiel Stillen. Also das Stillen ist ja auch so ein mega besetztes Thema, finde ich. Eigentlich sagen alle oder viele, ja es ist gut. Aber es ist nur gut für eine gewisse Zeit. Solange man länger stillt als acht, neun, zehn Monate, wird man schon komisch angekuckt auch unter Freunden oder Freundinnen. Alles hat so eine gewisse Zeit und ab dann bricht der Diskurs oder Einstellung, auch mit Leuten, mit denen man sehr viel teilt, und man wird schräg angekuckt. Das ist mir vorher nie so passiert in anderen Themenbereichen vor meiner Elternschaft.“ (Annika, Absatz 74)

Am Beispiel des Stillens konkretisieren sich normative Vorgaben zur Handlungsanweisungen. Muttersein wird so zu einer Gratwanderung zwischen Übermutter 
und Rabenmutter, zwischen ,zu egoistisch`oder ,zu selbstaufopfernd ‘, aber nicht zu einem Ort der subjektiven Selbstbestimmung.

Dennoch lassen sich in den Interviews auch Bemühungen erkennen, sich von gesellschaftlichen Normen abzugrenzen und sich gegen den normativen Druck zu stellen:

„Und ich hab’ mich dann total verabschiedet, nein, das stimmt auch wieder nicht. Aber irgendwie jetzt, bei meiner zweiten Tochter, war mir einfach viel wichtiger, diese ganz persönlichen Sachen zu beachten, als jetzt irgendeinem Bild hinterher zu rennen, was ich mir vorstelle, wie man als Mutter zu sein hat.“ (Marion, Absatz 34)

Marion ist entschieden, sich von einem normativen Mutterbild abzugrenzen und ahnt zugleich, dass dies nicht ganz gelingt.

Zusammenfassend lässt sich festhalten, dass eine selbstbestimmte Positionierung gegenüber den normativen Vorgaben nicht so ohne Weiteres möglich ist. Damit einhergehende Dilemmata lassen sich von den Müttern nicht einfach individuell lösen, es bleiben Zweifel, Selbstkritik und Selbstwertprobleme. Viele Mütter thematisieren einen ,Druck von allen Seiten', der sich sowohl in der subjektiven Bewertung des eigenen Handelns wie im privaten Umfeld, seitens von Freund*innen oder Arbeitskolleg*innen, bemerkbar macht. Besonders prominente ,Schauplätze“ der Auseinandersetzung sind der Zeitpunkt des Wiedereinstiegs in die Berufstätigkeit, das Stillen sowie die Betreuungsfrage. Die Frage des Kindeswohls spielt für alle Bereiche eine zentrale Rolle. Im Kontrast zum Begründungszusammenhang der Wahlfreiheit wird hier ein (innerer) Konflikt deutlich, der den Handlungsspielraum der Mütter unmittelbar beeinflusst.

\subsection{Der Kindeswohldiskurs}

Das Wohlergehen des Kindes spielt in allen Interviews eine entscheidende Rolle und beeinflusst die innerfamiliäre Arbeitsteilung. Linda:

„Ich würde gerne in Teilzeit zurückgehen erstmal, um am Anfang halt nicht eben so spät oder nicht so lang in die Krippe zu lassen, weil ich finde, für so ein kleines Kind ist es einfach lang. Ein ganzer Tag in der Krippe und unter fremden Leuten, auch wenn sich dann irgendwann gewöhnt und schlafen und alles und weg von den Eltern, ist ein bisschen schade. Weil, ich sag jetzt mal, man will ja nicht Kinder bekommen, um sie abzugeben.“ (Linda, Absatz 5)

Während ihr Wunsch nach Teilzeit - verknüpft mit der Vorstellung, kleine Kinder nicht so lange in die Krippe „unter fremde Leute“ geben zu wollen - zunächst den Fokus auf das Kind richtet, so verweist ihre Schlussfolgerung, dass ,man ja nicht 
Kinder bekommen will, um sie abzugeben“, zugleich auf die Wirkmächtigkeit entsprechender normativer Setzungen. Diese sind vom gesellschaftlich sehr präsenten und durch bestimmte Normen geprägten Kindeswohldiskurs beeinflusst. Obwohl Linda hier geschlechtsneutral von „Eltern“ spricht, bezieht sie Vorstellungen über eine gute kindliche Entwicklung nur auf sich als Mutter. Für ihren Partner, der Vollzeit arbeitet und durch diesen Anspruch eigentlich ebenso normativ angerufen wird, scheint der Kindeswohldiskurs andere Konsequenzen zu haben und wird dezidiert nicht auf eine Handlungsebene übertragen, die seine Berufstätigkeit tangiert.

Einen weiteren Aspekt betont Marlene, wenn sie ihr Handeln als Mutter daran orientiert, ihrem Kind später die bestmöglichen Chancen in der Gesellschaft zu ermöglichen. Marlene: „Ich bin halt jemand, der macht sich viele Gedanken, wie möchte ich mein Kind, wie möchte ich jetzt schon die Weichen für das spätere Leben quasi“" (Marlene, Absatz 63). Um ihr Kind optimal ,für das spätere Leben zu fördern', bedarf es nicht nur ausgewählter Erziehungsmethoden, sondern auch ihrer Präsenz. Hier taucht - wie bereits in der Zeitschriftenanalyse herausgearbeitet - als Begründung ein populärwissenschaftliches Verständnis von Bindungs- und Entwicklungspsychologie auf, das den späteren ,Erfolg“ des Kindes nicht nur in der Entwicklung der frühen Kindheit verortet, sondern in einem direkten Beziehungskontext mit der Mutter.

Auch bei den interviewten homosexuellen Müttern wird auf das Kindeswohl Bezug genommen, um die Aufteilung der Arbeiten in der Familie zu erklären. Rita:

„Gut, sie ist arbeiten gegangen, aber nur deswegen, weil sie einen Job hatte. Ich hatte keinen Job, also ich hätte vielleicht auch arbeiten gehen können für ein paar Stunden dann irgendwann nach einem Jahr, aber ich habe tatsächlich nicht aktiv gesucht und nicht eigentlich haben wollen, weil ich wollte diese Bachelorarbeit eigentlich fertig machen. Also an sich, es wirkt vielleicht traditionell, ist es aber eigentlich gar nicht wirklich so, weil schon allein, also es ist eigentlich wirklich alles wegen der Kinder.“(Rita, Absatz 21)

Ritas Einschätzung der innerfamiliären Aufteilung der Arbeit als „traditionell“ muss zwar vor dem Hintergrund der Gleichheitsnorm legitimiert werden; als Begründung wird das Anfertigen einer Abschlussarbeit angeführt. Abschließend dominiert aber die Aussage, dass „es eigentlich wirklich alles wegen der Kinder [ist]“.

So mündet Ritas Ringen um eine eindeutige Haltung in den Bezug auf das Kindeswohl und ermöglicht ihr so die gewählte Arbeitsaufteilung mit der Gleichheitsnorm in Einklang zu bringen. Noch verständlicher wird das Argument ,wegen der Kinder" analog zu den befragten heterosexuellen Müttern unter der Prämisse, dass das Kindeswohl auch für sie als leibliche Mutter einen anderen und normativ weitaus zentraleren Stellenwert hat. Obwohl die Orientierung an gesellschaftlichen Normen und wissenschaftlichen Vorgaben vordergründig auf die subjektive Einstel- 
lung der Mutter verweist, zeigt der folgende Abschnitt, dass die Orientierung am Verhalten anderer Mütter dabei eine zentrale Rolle spielt.

\subsection{Andere Mütter in der Funktion als ,Spiegel‘}

Die weiter oben beschriebene Verschiebung des ,Vereinbarkeitsproblems ' von Erwerbs- und Familienarbeit auf das ,Vereinbarkeitsproblem` zwischen Mutterrolle und moderner, ,emanzipierter' Frau* bestimmt auch die Beziehungsdynamik unter Müttern. Andere Mütter werden als ,Spiegel' gebraucht, um das eigene Handeln als Mutter zu bestätigen - oder auch, um sich abzugrenzen. Die Selbstpositionierung als Mutter reinszeniert sich in der mütterlichen Peergroup auf verschiedene Weise. So beschreibt Annika:

„Es sind mehr implizite Nachfragen und natürlich auch, das Vorleben anderer Modelle sozusagen. (...) Naja, das sind halt Modelle, die sind sehr verschieden und wir können gar nicht so offen darüber reden, eigentlich auch, manchmal wie, wie wir gegenseitig unsere Modelle irgendwie finden, weil es so super privat ist, also es ist irgendwie so schnell auch das politisch und privat, beides irgendwie. Ich finde, meine, also unser Modell, genau, ist so schnell auch politisch irgendwie. Also es hat ja mit dem Ganzen zu tun, wie ist Arbeit aufgeteilt zwischen den Geschlechtern, wie ist es angesehen und so. Manchmal ist auch schwer darüber offen zu reden, weil sie mir natürlich nicht sagen kann, ich finde irgendwie rückständig, wie du lebst und dass du so viel zuhause hockst. Und ich kann ihr nicht sagen, ich finde nicht gut, dass keiner euer Kind betreut, der nicht auch mit eurer Familie zu tun hat, zum Beispiel. Das ist so schnell auch am Kern der Gefühle. Das Thema Familie halt, also.“ (Annika, Absatz 86)

Einen wesentlichen Konflikt im Austausch über Elternschaft und Mutterschaft erlebt Annika darin, dass „Modelle“ von Mutterschaft und Elternschaft zugleich privat und politisch seien, denn wie die Arbeit zwischen den Geschlechtern aufgeteilt würde, bewege sich immer auch nah ,am Kern der Gefühle“. Dadurch würde ein offenes Sprechen enorm erschwert und damit zugleich die Möglichkeit darüber nachzudenken, dass es bei der Wahl von Elternmodellen unausgesprochen auch um grundsätzliche Fragen elterlicher Kooperation gehe.

Beziehungen zwischen Müttern sind - neben dem Wunsch nach respektvollem Umgang mit den mütterlichen Unterschiedlichkeiten - noch mit weiteren Konfliktfelder verbunden. Dazu erneut Linda:

„Mir hat dieser Austausch [mit anderen Müttern] unheimlich gutgetan, einfach zu sehen, dass es anderen genauso geht, weil ich finde, bevor man ein Kind bekommt, kann man sich nicht vorstellen, wie anstrengend so etwas ist. Wie kräftezehrend diese Nächte sind. Man denkt sich halt immer, ich komme zum Krankenhaus raus, das Kind liegt auf der Couch, es schläft 
den GANZEN Tag. Ich kann putzen, bügeln, kochen, (...) Haushalt. Ich kann schlafen. Ich kann so eine Art machen, was ich will, weil das Kind schläft ja den ganzen Tag.“ (Linda, Absatz 7)

Hier wird deutlich, wie sehr Linda den Austausch mit anderen Müttern braucht, um sich mit ihren mütterlichen Erfahrungen sicher - und vermutlich auch ,normal ${ }^{\text {` }}$ - zu fühlen. Dieser Austausch kann auch Konflikte mit dem Partner/der Partnerin entschärfen und normalisieren:

„Ja, ja klar, weil man da ja auch hören möchte, dass auch andere Männer den Teller stehen lassen, oder solche Sachen. Das ist schon wichtig, ja. Auch wie die Männer sich in der Vaterrolle sehen oder in dieser Ernährer-Rolle. Das ist schon auch interessant zu sehen: ,Och, bei euch läuft das auch so'. Ihr seid vielleicht, ihr habt zwar vielleicht ein anderes Lebenskonzept oder seid ein anderer Charakter, Charaktere als wir. Im Grunde genommen sind diese Rollenklischees tatsächlich ja da. Die existieren und es ist nicht nur bei uns so. Nicht nur wir sind so, wie wir sind, sondern es läuft bei anderen auch so. Doch, das ist super wichtig." (Dita, Absatz 68)

Die Erfahrung, dass „Rollenklischees“ auch bei anderen Paaren wirksam sind, führt jedoch für Dita nicht dazu, gemeinsam mit den Müttern (also kollektiv) diese Klischees als Geschlechterstereotypien zu hinterfragen, sondern dient anscheinend lediglich der individuellen Entlastung.

Der Modus des Vergleichens gibt Müttern einerseits die Möglichkeit sich von Normen zu distanzieren, wenn diese auch von anderen Müttern nicht erfüllt werden. Andererseits können überfordernde Erfahrungen damit auch normalisiert werden. Der Modus des Vergleichens steht somit eng mit dem normativen Druck auf Mütter in Verbindung; zugleich kann er die eigene Identitätsposition als Mutter unterstützen.

Eine weitere Möglichkeit des Sich-Spiegelns in anderen Müttern liegt in der Abgrenzung von Müttern, die es scheinbar noch schwerer haben. Damit können eigene Konflikte und Unzufriedenheiten relativiert und normalisiert werden. Wo einerseits die mütterliche Peergroup als Stütze bei Erfahrungen von Überforderung dient, bewegt sich die erlebte Gemeinsamkeit in der Gruppe auf einem schmalen Grat und droht dort ins Gegenteil zu kippen, wo Mütter entweder andere Erfahrungen machen oder ein anderes ,Modell‘ leben. Das folgende Zitat von Linda verbindet die bisher beschriebenen Motive des Vergleichens und verdeutlicht, warum eine Solidarisierung unter Müttern so schwierig ist. Mütter, die es ,besser machen ‘ - in größerem Umfang erwerbstätig sind oder mehr Zeit mit dem Kind verbringen werden nicht nur als Konkurrenz wahrgenommen, sondern lösen Schuldgefühle und Versagensängste aus und greifen den eigenen Selbstwert als Mutter an. Linda: 
„Also ich hatte wirklich von guten Freundinnen echt gute Unterstützung, die mir auch gesagt haben, bei uns war das genauso. Und nicht so: ,Echt? Ne, kennen wir nicht. Wirklich? Ne. Weil das hätte ich am, das war, das hätte ich am wenigsten gebraucht. Eine Freundin, die so ein superperfektes Baby hat, was du auf die Couch legen kannst und die Kleine oder der Kleine stundenlang schläft und die gefühlt nebenbei alles machen können, weil das Kind ist ja das Brävste auf der ganzen Welt. Da habe ich zwar Freundinnen in Bayern. Zum Glück kamen die Babys erst wesentlich später zur Welt wie er, weil sonst hätte ich gefragt, was habe ich falsch gemacht.“ (Linda, Absatz 11)

Dass die Begegnung mit einer Freundin, die ein ,perfektes Baby“ hat, als Bedrohung der eigenen Identität als ,guter Mutter' gefürchtet wird, offenbart idealisierte Ansprüche an eine harmonische und konfliktfreie Mutter-Kind-Beziehung. Vor dem Hintergrund ihrer ,Machbarkeit ${ }^{6}$ - , die Freundin hat es ja auch geschafft ${ }^{*}$ wird das Verhalten des Kindes zum Bewertungsmaßstab als ,gute Mutter'. Diese ,verdrehte' Perspektive problematisiert Barbara folgendermaßen:

„Wenn ich Mütter sehe, die unglaublich viel sich mit ihrem Kind dort beschäftigen und die ganze Zeit mit denen so spielen im Sand, die ganze Zeit, und da merke ich, okay, jetzt sitze ich die ganze Zeit und genieße die Sonne und fühle mich gut und meinem Kind geht es eigentlich gut, aber ich habe ein schlechtes Gewissen, weil ich diese andere Frau sehe und denke: ,Oar, jetzt beschäftigt sie sich die ganze Zeit mit ihrem Kind, was denkt die wohl, was ich für eine bin?““(Barbara, Absatz 38)

Entgegen ihrem eigenen Gefühl kommen bei Barbara Selbstzweifel auf, wenn sie sich mit den Augen anderer Mütter betrachtet, die ,sich die ganze Zeit mit ihrem Kind" beschäftigen. Eigentlich weiß Barbara, dass es ihr und dem Kind in dem Moment gut geht, aber durch den Blick der anderen Mütter - ob real oder phantasiert - gerät die positive Bewertung ihres Verhaltens ins Wanken.

An den beschriebenen Strategien des Vergleichs mit anderen Müttern zeigt sich zweierlei: Einerseits wird deutlich, wie moralisierende und normativ aufgeladene Diskurse des Kindeswohl dazu beitragen, einen offenen und selbstbestimmten Austausch unter Müttern zu verhindern. In Kombination mit der Figur der mütterlichen Selbstbestimmung wird Selbstverantwortung nicht nur in Schuld verkehrt, sondern führt vielfach zu einer schmerzhaft erlebten Identitätsverunsicherung. Eine immer wiederkehrende Strategie diese abzuwehren ist es, andere Mütter abzuwerten oder zu meiden. Die diskursive Figur der Do-it-all Mother als Bezugspunkt individueller Selbstbestimmung legitimiert nicht nur Konkurrenz und Entsolidarisierung unter Müttern, sondern verhindert auch, die eigene Eingebundenheit in soziokulturell geprägte Konstruktionen von , guter Mutterschaft` zu erkennen.

Im persönlichen Kontakt - und zugleich über normative Diskurse hervorgerufene - Verunsicherungen und Ängste, etwas falsch zu machen, werden über den Pro- 
zess des, othering ' auf andere projiziert. Die Form des othering trägt hier dazu bei, dass affektive Zustände von Unzufriedenheit, Angst vor Bewertung und Scham nicht mehr vor sich selbst und anderen Müttern eingestanden werden müssen. Die psychische Dynamik des othering ermöglicht zwar vorübergehend eine bestimmte Form von Selbstbestätigung und auch Entlastung, geht aber gleichzeitig mit Rückzug einher und verstärkt die eigene Isolierung. Denn andere Mütter werden in dieser Logik zu potenziellen und bedrohlichen Konkurrentinnen, deren bewertender Blick gemieden werden muss.

Der Ausgangspunkt dieser Spaltungsbereitschaft liegt nicht in den psychischen Besonderheiten der Mütter, sondern in der Kombination einer Rhetorik der Selbstverantwortung und Wahlfreiheit mit normativen und moralisierenden Diskursen. Erst vor diesem Hintergrund kann eingeordnet werden, wieso in den Interviews die Angst vor Bewertung, insbesondere in Bezug auf andere Mütter, immer wieder ein zentrales Thema ist.

\section{FAZIT}

Sowohl in den Elternzeitschriften als auch in den Interviews wurde deutlich, dass Familie einen hochbrisanten und affektiv umkämpften Lebensbereich darstellt, an dem eine Vielzahl von Normen sowie Rollen- und Familienbilder verhandelt werden. Dabei ist die Frage der, Vereinbarkeit‘ von Familie und Beruf sowohl politisch wie ,privat' fester Bestandteil von Mutterschafts- und Elternschaftsdiskursen. Insbesondere über die Gestaltung der Mutter-Kind-Beziehung, das Stillen, über Betreuungsfragen sowie den Zeitpunkt des beruflichen Wiedereinstiegs werden normative Erwartungen an mütterliche Verhaltensweisen verhandelt.

In den Interviews zeigte sich besonders eindrücklich, dass die Themen Selbstverantwortung, Wahlfreiheit und Individualisierung den Begründungshorizont bilden, vor dem die Aussagen und Argumente der Mütter Bedeutung gewinnen. Wie bereits in der Analyse der Zeitschriften, realisiert sich auch hier eine paradoxe Gleichzeitigkeit zwischen der Gleichheitsnorm und geschlechtsspezifischen $\mathrm{Zu}-$ schreibungen an Mütter wie Väter. Die widersprüchlichen und hohen Erwartungen an Mütter sowie deren Selbstzuschreibungen führen mit Blick auf die ,Vereinbarkeit ${ }^{\star}$ von Erwerbs- und Familienarbeit zu einem hohen inneren Druck. Finanzielle Abhängigkeit vertieft zudem Selbstwertprobleme, wenn eine - zumindest rhetorische - Umdeutung der Mutterrolle zur Rolle der ,Familienmanagerin' nicht gelingt. Die Professionalisierung der Mutterrolle (sowohl in den Interviews wie in den Zeitschriften) spielt hierbei eine ambivalente Rolle, indem sie einerseits einen Gegendiskurs zu der Geringschätzung von Familienarbeit entwirft, andererseits jedoch dazu beiträgt, geschlechterstereotype Zuweisungen weiterhin zu verschleiern. 
Ein deutlicher Bezugspunkt für die Befragten ist die Rhetorik der Wahlfreiheit. Paradoxerweise führt gerade diese dazu, dass eine geschlechterstereotype Aufgabenteilung in der Familie sowie finanzielle Abhängigkeiten als individuelle Entscheidung verhandelt werden und Fragen der Geschlechterhierarchie nicht mehr auftauchen. Unter dem Diktum der Wahlfreiheit erscheinen geschlechterstereotype Anrufungen nicht mehr als Zwang, sondern können als selbst gewähltes Modell gepriesen werden. Damit verlagert sich die Zuschreibung von Verantwortung für die familiale Lebensgestaltung, das Wohlergehen des Kindes, aber auch das Engagement des Vaters sowie die unbezahlte Familienarbeit explizit auf die Mutter. Der postfeministische Diskurshorizont von Selbstbestimmung, Individualisierung und Wahlfreiheit trägt gerade auch auf diese Weise zu einer De-Thematisierung der strukturellen Geschlechterhierarchie bei.

Die in den Elternzeitschriften wie auch in den Interviews wirksamen heteronormativen Zuschreibungen insbesondere an Mütter rekurrieren auf ein tief in der Gesellschaft verankertes Geschlechterwissen. Hierbei sind Vorstellungen von Geschlecht und Familie in einen naturalisierten und biologistischen Zusammenhang gestellt. Im Kontext der Idealisierung von Mutterschaft und Familie spielt eine Adressierung von Frauen* insbesondere aus der Mittelschicht als selbstbestimmte und beruflich erfolgreiche Mütter eine zentrale Rolle. Die Affirmation einer gelungenen Mutterschaft bzw. Familienentwicklung als Ausdruck einer Identifikation mit neoliberalen Wertmaßstäben dient zugleich als Kontrastfolie zu ,anderen‘ Müttern

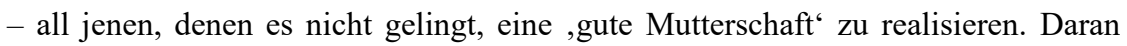
sind rassistische und klassistische Perspektiven auf ,Andere' (wie bspw. Alleinerziehende, ,Unterschichtmütter ${ }^{6}$ oder ,muslimische Mütter ${ }^{\star}$ ) unmittelbar anschlussfähig.

Unsere Analyse zeigt sehr eindrücklich, wie Geschlecht in Bezug auf die Versorgung von Kindern und die Strukturierung von Familie in Anschlag gebracht wird. Dadurch ergeben sich Anknüpfungspunkte an antifeministische Argumentationen zur Konstruktion von Familie, ohne dass der Impetus der Ideologisierung sofort deutlich wird.

Dabei nimmt der Kindeswohldiskurs sowohl in den Elternzeitschriften als auch in den Interviews eine zentrale Rolle ein und ermöglicht Anschlussstellen an die „Figur des bedrohten Kindes“ (Schmincke 2015) in antifeministischen Diskursen. Der Kindeswohldiskurs und die ihm inhärente Figur des ,hilflosen und schutzbedürftigen Kindes` ist eng mit normativ aufgeladenen Forderungen an Eltern, insbesondere an Mütter, verbunden. Damit einhergehende Begründungszusammenhänge bieten einerseits Antworten auf Erziehungsfragen; andererseits bietet die MutterKind-Beziehung eine besondere Angriffsfläche für normalisierende und biologistische Bilder von Mutterschaft. Eine derartige Verknüpfung von ideologisierenden Mutterschaftskonzepten und Kindeswohl ist nicht neu und lässt sich bis in das 19 . Jahrhundert zurück nachzeichnen. Auch wenn sich das Wissen um die Entwicklung 
des Kindes seither deutlich verändert hat, bleiben bestimmte Überzeugungen hartnäckig bestehen und beschreiben Kindheit sowie Kindesfürsorge, Elternschaft und Erziehungsanforderungen mit vermeintlich universal gültigen Vorstellungen. Gerade weil sie das Private, Intime und Alltägliche der Menschen durchziehen, stellen die Vorstellungen von Mutterschaft ein wichtiges Terrain dar, auf dem Diskurse auf subtile, aber umso wirkmächtigere Weise Einfluss nehmen.

Die von McRobbie (2015) für Großbritannien beobachtete Entwicklung, dass die Bezugnahme auf feministische Topoi an einer Neudefinition von Alltagsthemen ansetzt, wird durch unsere Ergebnisse gestützt. Wichtig erscheint dabei McRobbies Erkenntnis, dass der Postfeminismus seine Überzeugungskraft nicht primär aus einem Rückbezug auf konservative Rollenmodelle bezieht, sondern gerade aus seiner Fähigkeit, eine Synthese aus bestimmten Lesarten wissenschaftlicher Erkenntnisse mit neoliberalen Identitätsangeboten einzugehen. Hier zeigen unsere Analysen, wie über eine Rhetorik der Selbstbestimmung konservative Vorstellungen und Positionen von Mutterschaft mit modernen mütterlichen Anrufungen verknüpft werden. In der von uns herausgearbeiteten Figur der Do-it-all Mother wird der Anspruch ,alles zu schaffen' zu einem einflussreichen diskursiv-wirksamen Maßstab und nimmt Bezug auf eine Psychologie des Selbst, das Konzepte wie Empowerment und Selbstfürsorge in den Dienst neoliberaler Erfolgszwänge stellt (Rutherford 2018). Die postfeministische Behauptung, dass Frauen* bereits autonome, aktive und selbstbestimmte Subjekte sind (Rutherford 2018; McRobbie 2009), verschiebt die Realisierung des Emanzipationsanspruchs auf die individuelle Ebene und verdeckt den Zusammenhang mit gesellschaftlichen Strukturen. Der selektive Pluralismus und somit auch Diskursstrukturen wie rhetorische Modernisierung (Wetterer 2003) sowie affirmative Assimilation (Tichy/Krüger-Kirn 2020) üben eine stabilisierende Wirkung auf hierarchische Geschlechter- und Elternpositionen aus. Hier wird die postfeministische Vereinnahmung feministischer Politiken und Errungenschaften (vgl. Gill 2016; McRobbie 2009, 2015) besonders deutlich.

,Feminismus' wird in diesem Verständnis nicht mehr als ein politisches Projekt für die Unterstützung von Frauen*- und Mütterinteressen verstanden, sondern als Anrufung und Anforderung an Mütter, selbstbestimmt und finanziell unabhängig zu sein - eine Anrufung, die Mütter im Zweifel eher unter Druck setzt als ihnen Unterstützung zu bieten.

Die scheinbare Affirmation feministischer Errungenschaften erfolgt bei gleichzeitigem Unsichtbarmachen feministischer Herrschaftskritik. Über eine solche Umschreibung des Feminismus, die sich in besonderer Weise auf die Verzahnung von neoliberalem Feminismus und konservativen Familienideologien stützt, gelingt es gegenwärtig, feministische Themen wie Selbstbestimmung und Mutterschaft aus ihren herrschaftskritischen Bezügen zu lösen und in postfeministische Diskurse einzupassen. Auf diese Weise können emanzipatorische Errungenschaften im Bereich der Geschlechterverhältnisse, der Sexualität sowie der Familie und die damit ein- 
hergehenden sozialen wie kulturellen Veränderungen von Gesellschaft unsichtbar gemacht und - ohne offensichtlichen autoritären Druck - zurückgenommen werden.

Die versteckte Geschlechteragenda des Postfeminismus ist von einer tiefen Paradoxie gekennzeichnet, die dazu führt, dass eine Politisierung, d.h. das Sichtbarmachen und Hinterfragen struktureller und gesellschaftlich bedingter Ungleichheiten und Diskriminierungen erschwert wird. In diesem Zusammenhang ist unsere Analyse, dass sich das ,Vereinbarkeitsproblem` auf einen Konflikt zwischen Mutterrolle und der Rolle als emanzipierter Frau* ausgeweitet hat, von besonderer Tragweite. Hier verkehrt sich die Figur der mütterlichen Selbstbestimmung in einen Diskurs von Schuld (im Kontext moralischer Deutungshoheit über Mutterschaft) und begünstigt eine ,Spaltungsbereitschaft' unter Müttern. Erst vor dem Hintergrund der Indienstnahme von Selbstverantwortung und Wahlfreiheit für die Durchsetzung normativer Vorstellungen von Mutterschaft wird verständlich, wieso der Vergleich mit anderen Müttern meist mit vorweggenommenen Schuldzuweisungen einhergeht. Dies verschärft Konkurrenz und Entsolidarisierung unter Müttern und verhindert die Wahrnehmung kollektiver Ungleichheitserfahrungen. Die dadurch bedingte Entsolidarisierung kann als zentrales Ergebnis unserer Analysen gelten. Die weitreichende Relevanz dieses Befundes liegt in der Erkenntnis, dass der postfeministische Fokus auf Selbstbestimmung und Wahlfreiheit an die Stelle von gemeinsamen Anliegen und Solidarität tritt. Die damit entstehende Leerstelle und Erfahrung der Isolation kann dann potenziell von antifeministischen Diskursen besetzt und instrumentalisiert werden.

Um hinter dem neoliberalen Diskurs von Selbstverantwortung und Wahlfreiheit konservative Politiken und strukturelle Ungleichheiten im Geschlechterverhältnis in ihren gegenwärtigen Gestalten sichtbar zu machen, ist eine Reflexion sich wechselseitig bedingender Zusammenhänge zentral. In antifeministischen Diskursen über Familie und Kindeswohl wird jedoch die Trennung genau jener Zusammenhänge in ideologischer Absicht vollzogen. Die affektive Heftigkeit, die dabei spürbar wird, unterstreicht die Annahme, dass es dabei um mehr geht als um Gender Trouble auf der Ebene vergeschlechtlichter Identitäten - nämlich um strukturelle Fragen von Geschlecht und gesellschaftlichen Machtverhältnissen.

\section{LITERATUR}

Adam, Barry D. (1995): The Rise of a Gay and Lesbian Movement. Revised edition, New York: Twayne Publishers.

AWA (Allensbacher Markt- und Werbeträgeranalyse) (o.J.): Printmedien. [https: //www.ifd-allensbach.de/awa/medien/printmedien.html; abgerufen am 01.01. 2020; auf der Seite sind immer nur die Zahlen des Vorjahres abrufbar]. 
BAG (Bundesarbeitsgemeinschaft kommunaler Frauenbüros und Gleichstellungsstellen) (Hg.) (2018): Antifeminismus als Demokratiegefährdung?! Gleichstellung in Zeiten von Rechtspopulismus. [https:/www.frauenbeauftragte.org/sites/ default/files/uploads/downloads/antifeminismus_als_demokratiegefaehrdung.pd f; abgerufen am 01.04.2020].

Birsl, Ursula (2020): „Paradoxien und Aporien des Antifeminismus. Eine demokratietheoretische Einordnung“, in: Henninger/Birsl, Antifeminismen. ,Krisen“Diskurse mit gesellschaftsspaltendem Potential?, Bielefeld: transcipt.

Bowlby, John (1973): Mütterliche Zuwendung und geistige Gesundheit, München: Kindler.

Bowlby, John (1975): Bindung. Eine Analyse der Mutter-Kind-Beziehung, München: Kindler.

BMFSFJ (Bundesministerium für Familie, Senioren, Frauen und Jugend) (Hg.) (2006): Familie zwischen Flexibilität und Verlässlichkeit. Perspektiven für eine lebenslaufbezogene Familienpolitik. [https://www.bmfsfj.de/blob/jump/76276/7 --familienbericht-data.pdf; abgerufen am 25.04.2016].

BMFSFJ (Bundesministerium für Familie, Senioren, Frauen und Jugend) (2012): Familienreport 2012. Leistungen, Wirkungen, Trends, Paderborn: Bonifatiusdruck GmbH.

BMFSFJ (Bundesministerium für Familie, Senioren, Frauen und Jugend) (2017): Zweiter Gleichstellungsbericht der Bundesregierung. [https:/www.gleichstel lungsbericht.de/de/topic/2.zweiter-gleichstellungsbericht-der-bundesregierung. html; abgerufen am 21.06.2017].

Bundesinstitut für Bevölkerungsforschung (Hg.) (2017): Familienleitbilder. Alles wie gehabt? Partnerschaft und Elternschaft in Deutschland, Wiesbaden.

Derboven, Wibke (2019): Elternschaft als Arbeit: Familiales Care-Handeln für Kinder. Eine arbeitssoziologische Analyse, Bielefeld: transcript.

Diabaté, Sabine (2015): „Mutterleitbilder heute. Zwischen Autonomie und Aufopferung", in: Bevölkerungsforschung Aktuell 3/2015, S. 2-8.

Dolderer, Maya/Holme, Hannah/Jerzak, Claudia/Tietge, Ann-Madeleina (Hg). (2016): O Mother, Where Art Thou?, Münster: Westfälisches Dampfboot.

Fröhlich, Milan/Hellwig, Marius/Spicker, Rachel (2018): „Frauen im rechtspopulistischen und rechtsextremen Spektrum“, in: BAG, Antifeminismus als Demokratiegefährdung?! Gleichstellung in Zeiten von Rechtspopulismus, S. 17.

Gerhard, Ute (2012): Frauenbewegung und Feminismus. Eine Geschichte seit 1789, Nördlingen: Beck.

Gill, Rosalind (2016): „Post-Postfeminism? New Feminist Visibilities in Postfeminist Times“, in: Feminist Media Studies 16, S. 610-630.

Hark, Sabine/Villa, Paula-Irene (Hg.) (2015): Anti-Genderismus. Sexualität und Geschlecht als Schauplätze aktueller politischer Auseinandersetzungen, Bielefeld: transcript. 
Henninger, Annette (2020): „Antifeminismen. ,Krisen“-Diskurse mit gesellschaftsspaltendem Potenzial?“”, in: Henninger/Birsl, Antifeminismen. ,Krisen`-Diskurse mit gesellschaftsspaltendem Potential?, Bielefeld: transcipt.

Jäger, Margarete (2008): „Diskursanalyse: Ein Verfahren zur kritischen Rekonstruktion von Machtbeziehungen“, in: Ruth Becker/Beate Kortendiek (Hg.), Handbuch Frauen- und Geschlechterforschung, Wiesbaden: Springer VS, S. 378-383.

Jäger, Siegfried (1993): Kritische Diskursanalyse. Eine Einführung, Duisburg: DISS.

Kemper, Andreas. (2014): Keimzelle der Nation? Familien- und geschlechterpolitische Positionen der AfD. Eine Expertise. Friedrich-Ebert-Stiftung. [http:// library.fes.de/pdf-files/dialog/10641-20140414.pdf; abgerufen am 01.01.2020].

Kemper, Andreas (2016): „Geschlechter- und familienpolitische Positionen der AfD“, in: Wolfgang Kastrup/Helmut Kellershohn (Hg.), Rechte Wut- und Hassbürger im Kulturkampf. Rechtsruck in Deutschland, Münster: Unrast-Verlag, S. 147-160.

Klaus, Elisabeth (2016): „Antifeminismus und Elitefeminismus. Eine Intervention“, in: Feministische Studien 26, S.176-186.

Koppetsch, Cornelia/Speck, Sarah (2015): Wenn der Mann kein Ernährer mehr ist. Geschlechterkonflikte in Krisenzeiten, Berlin: edition suhrkamp.

Krüger-Kirn, Helga (2017): „Elternschaft und Gendertrouble“, in: Heike Schnoor, (Hg.), Psychosoziale Entwicklung in der Postmoderne, Gießen: PsychosozialVerlag, S. 215-230.

Krüger-Kirn, Helga/Näser-Lather, Marion/Lather, Dietger/Schumacher, Nina (2019): „Strategien zur Manipulation der Öffentlichkeit. Unwissenschaftliche Kritik an ,Gender" in Ulrich Kutscheras Gender-Paradoxon“, in: Marion NäserLather/Anna Lena Oldemeier/Dorothee Beck (Hg.), Backlash?! Antifeminismus in Wissenschaft, Politik und Gesellschaft, Sulzbach/Taunus: Ulrike Helmer, S. 128-148.

Lang, Juliane (2017): „Feindbild Feminismus. Familien- und Geschlechterpolitik in der AfD“, in: Stephan Grigat (Hg.), AfD und FPÖ. Antisemitismus, völkischer Nationalismus und Geschlechterbilder, Baden-Baden: Nomos, S. 61-78.

Lenz, Ilse (2013): „Geschlechterkonflikte um die Geschlechterordnung im Übergang: zum neuen Antifeminismus“, in: Erna Appelt/Brigitte Aulenbacher/Angelika Wetterer (Hg.), Gesellschaft: feministische Krisendiagnose, Westfälisches Dampfboot, S. 204-226.

Lessenich, Stephan (2008): Die Neuerfindung des Sozialen. Der Sozialstaat im flexiblen Kapitalismus, Bielefeld: transcript.

Maihofer, Andrea/Schutzbach, Franziska (2015): ,Vom Antifeminismus zum ,AntiGenderismus “ - Eine zeitdiagnostische Betrachtung am Beispiel Schweiz“, in: Hark/Villa, Anti-Genderismus. Sexualität und Geschlecht als Schauplätze aktueller politischer Auseinandersetzungen, S. 201-217. 
Maierhofer, Sara/Strasser, Irene (2016): „Das war bei uns ganz einfach, weil ich war der Verdiener. Elterliche Arbeitsteilung im Fokus von Traditionaliserungsprozessen“, in: Journal für Psychologie 24, S. 225-252.

Marga, Vicedo (2013): The Nature and Nurture of Love: From Imprinting to Attachment in Cold War America, Chicago/London: University of Chicago Press.

McRobbie, Angela (2009): The Aftermath of Feminism. Gender, Culture and Social Change, London: Sage.

McRobbie, Angela (2015): „Das Geschlecht des Postfordismus: passionate work, ,Risikoklasse“ und ,eigenes Leben““, in: Katharina Walgenbach/Anna Stach (Hg.), Geschlecht in gesellschaftlichen Transformationsprozessen, Opladen/ Berlin/Toronto: Barbara Budrich, S. 51-73.

Menke, Katrin (2017): „Eltern als ,Wirtschaftssubjekte“? Die selektiven Folgen einer ökonomisierten Familienpolitik auf die Wahlfreiheit von Müttern und Vätern“, in: Annette von Alemann/Sandra Beaufays/Beate Kortendiek (Hg.), Alte neue Ungleichheiten? Auflösungen und Neukonfigurationen von Erwerbs- und Familiensphäre, Opladen: Barbara Budrich, S. 42-58.

Metzger, Hans-Geert/Dammasch, Frank (Hg.) (2017): Männlichkeit, Sexualität, Aggression. Zur Psychoanalyse männlicher Identität und Vaterschaft, Gießen: Psychosozial Verlag.

Rinken, Barbara (2012): Gender- und Familienbilder in Elternratgebern. Heterogene Sichtweisen auf Kindheit und Familie. Ergebnisbericht einer Diskursanalyse, München: Deutsches Jugendinstitut.

Rutherford, Alexandra (2018): „Feminism, Psychology and the Gendering of Neoliberal Subjectivity. From Critique to Disruption“, in: Theory \& Psychology 28, S. 619-644.

Scheele, Sebastian (2015): „Das trojanische Zombie-Pferd. Fünf Thesen zu einer diskursiven Verschiebung im gegenwärtigen Antifeminismus“, in: Friedrich Burschel (Hg.), Aufstand der ,Wut-bürger'. AfD, christlicher Fundamentalismus, Pegida und ihre gefährlichen Netzwerke, S. 32-46. [https://www.rosalux. de/publikation/id/8319/aufstand-der-wutbuerger/; abgerufen am 01.01.2020].

Schmincke, Imke (2015): „Das Kind als Chiffre politischer Auseinandersetzung am Beispiel neuer konservativer Protestbewegungen in Frankreich und Deutschland“, in: Hark/Villa, Anti-Genderismus. Sexualität und Geschlecht als Schauplätze aktueller politischer Auseinandersetzungen, S. 93-107.

Smith, Anna M. (1994): New Right Discourse on Race and Sexuality, Cambridge: Cambridge University Press.

Sommerfeld, Denise P. (1989): „The Origins of Mother Blaming: Historical Perspectives on Childhood and Motherhood“, in: Infant Mental Health Journal 10, S. 14-24.

Statistisches Bundesamt (2019): Kinderlosigkeit, Geburten und Familien - Ergebnisse des Mikrozensus 2018. [https://www.destatis.de/DE/Themen/GesellschaftUmwelt/Bevoelkerung/Haushalte-Familien/Publikationen/Downloads-Haushal 
te/geburtentrends-tabellenband-5122203189014.pdf?_blob=publicationFile; abgerufen am 11.05.2020].

Thiessen, Barbara/Villa, Paula-Irene (2009): „Mütter und Väter: Diskurse - Medien - Praxen. Eine Einleitung“, in: Dies. (Hg.), Mütter - Väter: Diskurse, Medien, Praxen, Münster: Westfälisches Dampfboot, S. 7-21.

Tichy, Leila Zoë/Krüger-Kirn, Helga (2019): „The ,Do-It-All Mother“ - Discursive Strategies and Post-Feminist Alliances in Parenting Magazines“, in: Open Gender Journal. [DOI: doi.org/10.17169/ogj.2019.75].

Tichy, Leila Zoë/Krüger-Kirn, Helga (2020): „,Mama, Mami, Kind“ - Zur Diskursivierung homosexueller Elternschaft in Elternzeitschriften“, in: Gender Sonderheft (im Erscheinen).

Walters, Suzanna D. (2012): „The Kids Are All Right But the Lesbians Aren't: Queer Kinship in US Culture“, in: Sexualities 15(8), S. 917-933.

Wetterer, Angelika (2003): „Rhetorische Modernisierung: Das Verschwinden der Ungleichheit aus dem zeitgenössischen Differenzwissen“, in: Gurdun-Axeli Knapp/Angelika Wetterer (Hg.), Achsen der Differenz. Gesellschaftstheorie und feministische Kritik II, Münster: Westfälisches Dampfboot, S. 286-320.

\section{QUELLEN}

\section{Text-Sample Diskursanalyse}

Baby \& Familie

05.2010: Die neue Gelassenheit

07.2010: Die Macht der Hormone

10.2010: Die Gesundheits-Managerin

11.2010: Die neuen Väter

$02.2011 \mathrm{a}$ : Geht das wieder raus?

02.2011b: Mein tiefes inneres Wissen

03.2011: Die Macht der Väter

12.2011: Ein starker Start ins Leben

06.2012: Darf ich das?

07.2012: Putzen für Faulpelze

09.2012: Still-Strategie

04.2013a: Sind wir Opfer unserer Hormone?

04.2013b: Lasst uns endlich in Ruhe

02.2014: Zurück in den Job

05.2014: Ich liebe meinen Körper

08.2014: Stillen ohne Stress

12.2014: So bleibt die Liebe

01.2015: Gesund durchs erste Jahr 
09.2015: Von Anfang an stark!

11.2015: Mama in love

01.2016: Gutes Gefühl

03.2016: Auf ganz eigene Art

06.2016: Kollege Papa

07.2016: Stress mich noch mal

12.2016: Entspannt euch, Kinder!

02.2017: Zwischen Kind und Job

10.2017: „Ich bin nur noch die Putzfrau“

11.2017: Papa macht's anders!

12.2017: Der Zauber der ersten Stunde

\section{Eltern}

04.2010: Wann ist eine Mama eine Mama?

04.2011: Mama, Mami, Kind

05.2011: Bin ich eine emanzipierte Mutter?

03.2012: Das große Vater-Puzzle

06.2012: Ich bin eine Glucke

07.2012: Wenn nur die Mama trösten kann

09.2012: Was stört uns eigentlich an Jamie Lynne?

04.2013: Mein Baby aus dem Internet

06.2013: Wie teilen wir gerecht?

07.2013: Putzen mit Bauch

11.2013: Sind Väter heute zu mütterlich?

12.2013: Starke Mütter, tolle Väter

02.2014: Väter 2014. Zwischen Wunsch und Wirklichkeit

03.2014: Väter 2014. Zum Zuschauen verdammt

08.2014: Rooming-in: Wirklich rund um die Uhr?

11.2014: Ich bin Papa. Und eine Frau

06.2015: Konsequent!

10.2015: Vereinbarkeit für alle!

12.2015: Mehr Mutterschutz

04.2016: Jammer-Mamas, Depri-Daddys

05.2016: Bedauern, dass man Mutter geworden ist. Warum?

11.2016: Füttern für die Seele

02.2017: „Mein Modell wär's nicht“

05.2017: Mach du doch mal! - Nee, du!

08.2017a: Das Muttiversum

08.2017b: Von wegen Psychokram

12.2017: Liebe macht unsere Kinder groß 
Eltern special

2014: Danke

Nido

09.2010: Mythos Quality Time

11.2010: Selbst\&Ständig

02.2011: Immer Ich!

03.2011: Kreißsaalhelden

10.2011: Die bessere Hälfte

11.2011: Eine Frage der Balance

03.2012: Da wächst was

04.2012: Alles auf Anfang

05.2012: Vermisste Gefühle

07-08.2012: Auf Autopilot

09.2012: Darf ich mitspielen?

02.2013: Einatmen. Ausatmen.

04.2013a: Vater sein dagegen sehr

04.2013b: Das Beste fürs Kind

10.2013: Wir müssen anders arbeiten

11.2013: „Warum sollte irgendjemand alles haben können?“

03.2014: Und wo bleibe ich?

05.2014: Volle Kraft

10.2014: Familienbild

01.2015: Das Beste für mein Kind

05.2015: Entspannt unter Strom

07.2015a: Ganz der Vater?

07.2015b: „Die Frauenquote ist gut für Väter“

10.2015: Die Welt der Tandems

11.2015: „Endlich allein“

02.2016a: Achtsamkeit bringt Ruhe in den Alltag

02.2016b: „Frauen sehen sich als Zuverdienerinnen“

04.2016: Kontrolliert, manipuliert, abkassiert

09.2016: Er ist schwanger

01.2017: „Jemand muss verrückt nach mir sein“

02.2017: Der Mann im Vater

03.2017: Das nagt

09.2017: Das Leben hinter den Zahlen

\section{Interviews}

Interview Annika, geführt am 25.09.2018.

Interview Barbara, geführt am 19.02.2019. 
Interview Bettina, geführt am 15.03.2019.

Interview Dita, geführt am 07.02.2019.

Interview Lara, geführt am 27.11.2018.

Interview Linda, geführt am 05.11.2018.

Interview Marion, geführt am 03.09.2018.

Interview Marlene, geführt am 29.01.2019.

Interview Ramona, geführt am 16.10.2018.

Interview Rita, geführt am 14.03.2019. 